\title{
3-DIMENSIONAL LORENTZ SPACE-FORMS AND SEIFERT FIBER SPACES
}

\author{
RAVI S. KULKARNI \& FRANK RAYMOND
}

\section{Table of Contents}

\$1. Introduction . . . . . . . . . . . . . . . . . . . . . . 231

§2. Special features of the 3-dimensional Lorentz geometry of constant curvature $1 \ldots 235$

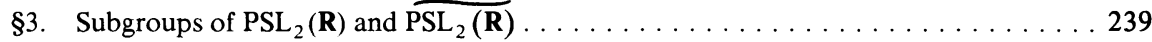

$\S 4$. Subgroups of $I_{0}\left(S_{\infty}\right)$, the level of a subgroup $\ldots \ldots \ldots \ldots \ldots \ldots \ldots \ldots \ldots$

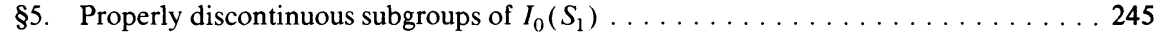

§6. Space-forms with solvable fundamental groups $\quad 247$

§7. Finiteness of Level . . . . . . . . . . . . . . . . . . . . . . . . . . . . . . . 248

§8. Standard Lorentz orbifolds and Seifert orbifolds . . . . . . . . . . . . . . . . 252

§9. Topology of standard orbifolds $\ldots \ldots \ldots \ldots \ldots \ldots \ldots \ldots \ldots \ldots \ldots \ldots \ldots$

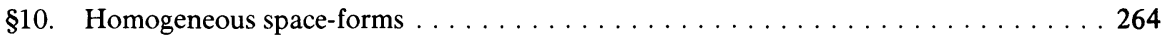

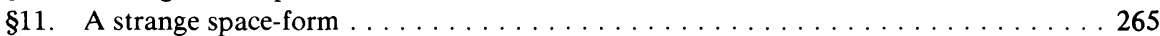

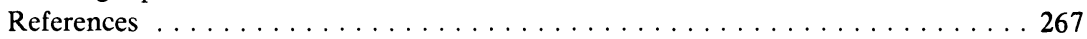

\section{Introduction}

A space-form is a complete pseudo-Riemannian manifold of dimension $\geqslant 2$ with constant curvature. A Lorentz space-form is a space-form with a Lorentz metric of signature $+--\cdots$. In this paper we study 3-dimensional Lorentz space-forms of constant curvature 1 , and unless there is a possibility of confusion, these will be often referred to simply as space-forms. The standard linear model for this geometry (the "3-dimensional anti-de Sitter space") is

$$
S^{1,2}=\left\{\left.(x, y)\left|x, y \in \mathbf{R}^{2},\right| x\right|^{2}-|y|^{2}=1\right\} \approx O(2,2) / O(1,2),
$$

cf. [38, p. 334]. This set-up differs markedly from the usual Riemannian set-ups in two respects: (1) the isotropy subgroup $O(1,2)$ is noncompact, so $O(2,2)$ does not act properly on $S^{1,2}$. This feature substantially restricts the discrete subgroups of $O(2,2)$ which can act properly discontinuously on $S^{1,2}$. (2) On

Received November 28, 1983 and, in revised form, January 10, 1985. The first author was partially supported by an NSF grant and a Guggenheim fellowship, and the second author by an NSF grant. 
the other hand, $S^{1,2} \approx S^{1} \times \mathbf{R}^{2}$ via the map $(x, y) \rightarrow(x /|x|, y)$, has $\pi_{1} \approx \mathbf{Z}$. So these space-forms can be constructed from the groups of isometries of the universal cover $\tilde{S}^{1,2}$. This feature allows for certain geometric realizations of an important class of 3-manifolds known as Seifert fiber spaces cf. [30], [20], [6, Chapter 12] of [7, Chapter VI]. This class of spaces has arisen naturally in widely different contexts cf. [27], [22], [23]. A reason for this, at least with hindsight, is that if in a mathematical problem a 3-manifold arises naturally and exhibits "interesting symmetries", it is likely to be a Seifert fiber space!

A more convenient model to study our problem is obtained by realizing that $-\frac{1}{8}$ (the Killing form) makes $\mathrm{PSL}_{2}(\mathbf{R})$ a Lorentz space-form. Write $P_{1}$ resp. $S_{1}$ for $\mathrm{PSL}_{2}(\mathbf{R})$ when considered as a Lie group resp. a space-form. Let $P_{n}$ resp. $S_{n}$ denote its $n$-fold connected covering space considered as a Lie group resp. a space-form, $1 \leqslant n \leqslant \infty$. Note that $S^{1,2}=S_{2}$ and $\tilde{S}^{1,2}=S_{\infty}$. A space-form $M$ has level $n$ if $n$ is the least integer, possibly infinite, such that $M$ is covered by $S_{n}$. To decide the level of a space-form is an important consideration in this problem. The full isometry group $I\left(S_{1}\right)$ is a certain extension of $P_{1} \times P_{1}$ by $\mathbf{Z}_{2} \times \mathbf{Z}_{2}$ and $I\left(S_{\infty}\right)$ can be realized as a certain extension of $\mathbf{Z}$ by $I\left(S_{1}\right)$, cf. $\S 2$. We construct a particular subgroup $J$ of $I\left(S_{\infty}\right)$ which acts properly on $S_{\infty}$. A discrete, torsion-free subgroup of $I\left(S_{\infty}\right)$ conjugate to a subgroup of $J$, and the corresponding space-form, for want of a better name, will be called standard. In recent years it has been found helpful to allow all properly discontinuous groups i.e. whose actions are not necessarily free, and the corresponding quotients, a là Thurston, are called orbifolds. The orbifolds corresponding to discrete subgroups of $I\left(S_{\infty}\right)$ which can be conjugated in $J$ will also be called standard. Analogously there is a purely topological notion of a Seifert orbifold which roughly speaking, is obtained as a quotient of a Seifert fiber space by a finite group of fiber-preserving homeomorphisms. Roughly speaking, a motivation of this paper was the realization that the discrete subgroups of $J$ are precisely a certain subclass of the "fundamental groups of Seifert orbifolds". In [8], cf. also [11, §7], this was proved in special cases by a differentialgeometric method.

Now we describe more precisely the contents of this paper. First we make a general investigation of subgroups of $I\left(S_{\infty}\right)$ and the ones among these which are discrete and among these the ones which act properly discontinuously on $S_{\infty}$. Since the identity component $I_{0}\left(S_{\infty}\right)$ is a certain quotient of $P_{\infty} \times P_{\infty}$, in the process, we have also obtained some information about subgroups of $P_{1}=\operatorname{PSL}_{2}(\mathbf{R})$ and its universal cover $P_{\infty}$. For example, we prove that a nonabelian free subgroup of $P_{\infty}$ contains an element which is in the image of the exponential map from the Lie algebra. We believe that these results should 
prove to be of interest in other contexts. One of our main results is

(5.2) Theorem. Let $\Gamma$ be a finitely generated torsion-free subgroup of $I_{0}\left(S_{1}\right)$ (which we identify with $P_{1} \times P_{1}$ ) such that $\Gamma$ acts properly discontinuously on $S_{1}$. Then there exists a discrete subgroup $\Phi$ of $P_{1}$ and a representation $\rho: \Phi \rightarrow P_{1}$ such that, up to switching of factors, $\Gamma=\{(x, \rho(x) \mid x \in \Phi\}$.

Among other things we classify solvable subgroups of $I\left(S_{\infty}\right)$ acting properly discontinuously on $S_{\infty}$. We have obtained criteria for a space-form to be of finite level e.g. this holds if the space-form is compact, cf. Theorem (7.2). This implies that a compact Lorentz space-form is finitely covered by a circle bundle over a closed orientable surface of genus $\geqslant 2$, and in a large number of cases the structure in fact must be a standard one, cf. (7.5). (For a long time we wondered whether every compact Lorentz space-form must be standard. But W. Goldman has recently come up with an argument showing for example that a standard structure on the tangent circle bundle over a closed surface of genus $\geqslant 2$ may be deformed to a nonstandard one!) A lemma which is crucially used for many of these results is that a subgroup $\approx \mathbf{Z}^{2}$ of $I_{0}\left(S_{1}\right)$ cannot act properly discontinuously on $S_{1}$, cf. (5.1). As for the standard space-forms we prove

Theorem. (a) (Structure). A standard Lorentz orbifold is an orientable Seifert orbifold with hyperbolic base, which, if closed, has nonzero Euler number.

(b) (Realization). Let $M$ be an orientable Seifert orbifold with hyperbolic base. Moreover if $M$ is closed assume that its Euler number is nonzero. Then $M$ admits the structure of a standard Lorentz orbifold.

(c) (Topology). A closed standard Lorentz orbifold is homeomorphic to either (i) a closed orientable Seifert fiber space with nonzero Euler number or (ii) a connected sum of lens spaces including $S^{3}$ and $S^{2} \times S^{1}$. Conversely all manifolds of types (i) or (ii) admit the structure of a standard Lorentz orbifold.

(d) (Volume). The volume of a closed standard Lorentz orbifold is an invariant of the underlying Seifert orbifold structure and is a rational multiple of $4 \pi^{2}$.

The proofs of these statements are in $\S 8$ and $\S 9$ where the notions of a Seifert orbifold and its Euler number are also made precise. Notice that the 3-manifolds of type (i) in the (Topology)-part are precisely the closed orientable Seifert fiber spaces not covered by (a closed surface) $\times S^{1}$.

We also classify homogeneous space-forms, i.e. ones with transitive isometry group. This corrects and partially completes the work of J. Wolf [37], [38] cf. also [10].

Added in proof. According to recent work of W. Goldman, a closed surface $\times S^{1}$ is never a Lorentz space-form, cf. (7.1.1). Consequently, using the 
theorem above, Proposition 7.5, §10.1, and [28], all closed Lorentz space-forms must be standard forms except possibly those forms homeomorphic to homogeneous forms. All the homogeneous forms, which are automatically standard, can be deformed, as W. Goldman shows, to nonstandard Lorentz space-forms. Therefore, all the closed Lorentz space-forms have been identified.

Finally we should mention a curious phenomenon in this Lorentz geometry which would never occur in any Riemannian case. There is a locally proper but not proper action of $\mathbf{Z}^{3}$ on $S_{\infty}$. The corresponding quotient must be considered as a "space-form" in the traditional sense of differential geometry. It is locally Hausdorff but globally non-Hausdorff. Topologically it is a torus bundle over a standard example of a non-Hausdorff manifold: the line with a double point!

We would like to point out to the reader, chiefly interested in the topology of 3-manifolds and who may wish to see as quickly as possible the connections between Seifert orbifolds and standard 3-dimensional Lorentz orbifolds, that it is possible to read $\S 8$ and $\S 9$ without studying the complete details treated in the earlier sections.

Our results concerning standard space-forms date back from 1977-1978. The standard space-forms are closely related to what Thurston has called the 3-manifolds with $\widetilde{\mathrm{SL}_{2}(\mathbf{R})}$-geometry cf. [34], ${ }^{1}$ geometry \#6. For Thurston, this geometry is any structure defined by a $J$-invariant Riemannian metric on $\overline{\mathrm{SL}_{2}(\mathbf{R})}=S_{\infty}$. The details of Thurston's work are yet to appear. P. Scott's recent work [31] gives an outline, and his theorem (4.15) has a close connection with part (a) of our theorem (8.5). As for the part (b) (realization) of (8.5) it appears from a description in a private communication to us by Thurston that his method of putting the $\overline{\mathrm{SL}_{2}(\mathbf{R})}$ structure on a class of Seifert fiber spaces is by explicitly constructing appropriate metrics. This method was used by one of us cf. [8] in special cases. The details along these lines for the whole class covered under part (b) of (8.5) would give a nice perspective on this problem. On the other hand, the method used here is based on a detailed understanding of the Lie groups involved, and has a different perspective. It may be remarked that from the Lie-as well as differential geometric-viewpoint this space-form problem is "dual" to the 3-dimensional spherical space-form problem, and the Lorentz geometry is a "natural" one, cf. $§ 2, \S 3, \S 9$.

A final remark: In this paper we have completely left out the natural question of deformations of Lorentz structures. We have discussed this theory in a more general context of deformations of geometric structures, in a later publication, cf. [14].

\footnotetext{
${ }^{1}$ Actually the group described in [34, p. 369] is not quite correct. It should be the group $J$.
} 
Notations. For any topological group $G$, its identity component is denoted by $G_{0}$, the center by $Z(G)$. If $H$ is a subgroup of $G, Z_{G}(H)$ resp. $N_{G}(H)$ denotes the centralizer resp. normalizer of $H$ in $G$. If $x \in G, i_{x}$ denotes the conjugation by $x$. Also $G \approx P \rtimes Q$ stands for " $G$ is isomorphic to a semidirect product of $P$ by $Q$ where $P$ is normalized by $Q$ ". An extension of a group $P$ by a group $Q$ means a group $G$ containing $P$ as a normal subgroup with $G / P \approx Q$. For an element

in $\mathrm{GL}_{2}(\mathbf{R})$,

$$
p=\left[\begin{array}{ll}
a & b \\
c & d
\end{array}\right]
$$

$$
p^{-}=\left[\begin{array}{ll}
a & b \\
c & d
\end{array}\right]^{-}
$$

denotes its image in $\mathrm{PGL}_{2}(\mathbf{R})$. Some more specialized notation is introduced in $\S 2$ and $\S 4$ which is used throughout the paper.

Acknowledgement. We owe special thanks to Bill Goldman, for taking interest in this work. He supplied (7.5), and showed the possibility of nonstandard deformations of certain standard compact Lorentz space-forms.

\section{Special features of the 3-dimensional Lorentz geometry of constant curvature 1}

(2.1). Let $P_{1}$ denote $\mathrm{PSL}_{2}(\mathbf{R})$. The center $Z\left(P_{1}\right)$ is trivial and the fundamental group of $P_{1}$ is isomorphic to $\mathbf{Z}$. Let $P_{n}, 1 \leqslant n \leqslant \infty$, denote the unique $n$-fold connected cover of $P_{1}$, which, as a group, is a central extension of $\mathbf{Z}_{n}$ by $P_{1}$. It is easily seen that $-\frac{1}{8}$ (the Killing form) induces a complete bi-invariant Lorentz metric with constant curvature 1 on all $P_{n}$ 's. We shall write $S_{n}$ for $P_{n}$ considered as a Lorentz manifold and $I\left(S_{n}\right)$ for its group of isometries. The action of $P_{n} \times P_{n}$ on $S_{n}$ via $(g, h) x=g \times h^{-1}$ is by Lorentz isometries since the metric is bi-invariant. The standard estimates for the dimensions of isometry groups show that the identity component $I_{0}\left(S_{n}\right)$ is isomorphic to $\left(P_{n} \times P_{n}\right) / Z_{n}$ where $Z_{n} \approx\left\{(z, z) \mid z \in Z\left(P_{n}\right)\right\}$. In particular $I_{0}\left(S_{1}\right) \approx P_{1} \times P_{1}$. The isotropy subgroup at the identity is given by

$$
\left\{(x, x) \mid x \in P_{n}\right\} / Z_{n} \approx P_{n} / Z\left(P_{n}\right) \approx P_{1} .
$$

The image of $(x, y) \in P_{\infty} \times P_{\infty}$ in $I_{0}\left(S_{\infty}\right)$ will be denoted by $[(x, y)]$.

We now describe the full isometry group $I\left(S_{n}\right)$. It has four components which geometrically correspond to "time"- and/or "space"-reversals. Consider a time-like resp. space-like vector as having positive resp. negative length.

(2.2) Proposition. Let $\sigma: A \rightarrow A^{-1}$ be the geodesic symmetry at the identity, and $i_{u_{0}}$ the lift of the conjugation of $P_{1}$ by

$$
u_{0}=\left[\begin{array}{cc}
1 & 0 \\
0 & -1
\end{array}\right]^{-}
$$


to $P_{n}$ fixing the identity. Then $\sigma$ preserves the space-orientation and reverses the time-orientation, and $i_{u_{0}}$ reverses both the time- and space-orientations. Moreover $\left\langle\sigma, i_{u_{0}}\right\rangle \approx \mathbf{Z}_{2} \times \mathbf{Z}_{2}$, the group $\left\langle I_{0}\left(S_{n}\right), i_{u_{0}}\right\rangle$ is the full group of orientationpreserving isometries, and $I\left(S_{n}\right)=\left\langle I_{0}\left(S_{n}\right), \sigma, i_{u_{0}}\right\rangle \approx I_{0}\left(S_{n}\right) \rtimes\left\langle\sigma, i_{u_{0}}\right\rangle$.

Proof. Both $\sigma$ and $i_{u_{0}}$ fix the identity and preserve the Killing form, hence are Lorentz isometries. On the Lie algebra level $\sigma$ acts by $A \rightarrow-A$ which clearly commutes with the action of $i_{u_{0}}$ on the Lie algebra. So $\left\langle\sigma, i_{u_{0}}\right\rangle \approx \mathbf{Z}_{2} \times$ $\mathbf{Z}_{2}$. Time-like and space-like subspaces at the identity in $P_{1}$ are given respectively by

$$
\begin{gathered}
C=\left\{\left[\begin{array}{cc}
c & s \\
-s & c
\end{array}\right]^{-} \mid c=\cos \theta, s=\sin \theta\right\}, \\
D=\left\{\left[\begin{array}{cc}
x & y \\
y & w
\end{array}\right] \mid x w-y^{2}=1, x>0\right\} .
\end{gathered}
$$

The latter may be coordinated by $(x, y) \in \mathbf{R}_{>0} \times \mathbf{R} \approx \mathbf{R}^{2}$. The map $i_{u_{0}} \mid$ (resp. $\left.\left.i_{u_{0}}\right|_{D}\right)$ has the form $\theta \rightarrow-\theta$ (resp. $(x, y) \rightarrow(x,-y)$ ) which shows that $i_{u_{0}}$ reverses both the time- and space-orientations, and so preserves the total orientation. Also the map $\left.\sigma\right|_{C}\left(\right.$ resp. $\left.\left.\sigma\right|_{D}\right)$ has the form $\theta \rightarrow-\theta$ (resp. $(x, y) \rightarrow$ $\left.(w,-y)=\left(\left(1+y^{2}\right) / x,-y\right)\right)$ which shows that $\sigma$ reverses the time-orientation and preserves the space-orientation. By lifting, the same assertions hold for all $P_{n}$ 's. Finally $I\left(S_{n}\right)$ has four components and $I_{0}\left(S_{n}\right) \cap\left\langle\sigma, i_{u_{0}}\right\rangle=\{e\}$ so the final assertion follows.

(2.3) Remark. There is another natural description of $i_{u_{0}}$. Consider $P_{1}$ as the group of orientation-preserving isometries of the hyperbolic plane $H$ which is taken as the upper half plane in $\mathbf{C}$. The map $z \rightarrow^{\psi}-\bar{z}$ is an orientationreversing isometry of $H$ and $I(H)=\left\langle P_{1}, \psi\right\rangle$. Also $P_{1}$ may be identified with $T_{1}(H)=$ the unit tangent bundle of $H$. The action of $\psi$ on $P_{1}$ by conjugation is the same as the one induced by $\psi$ on $T_{1}(H)$. From the Lorentz viewpoint a unit tangent circle is a time-like subspace and a section of the hyperbolic plane in $P_{1}$ is a space-like subspace, so $\psi$ acting on $P_{1}$ clearly reverses time- and space-orientations. One checks that indeed $i_{u_{0}}=\psi$ under identification of $P_{1}$ with $T_{1}(H)$.

(2.4). Since the isotropy subgroup is noncompact the action of $I\left(S_{n}\right)$ on $S_{n}$ is not proper. For the generalities on proper actions see [25] and [11, $\$ 1$ and the appendix]. So a discrete subgroup of $I\left(S_{n}\right)$ does not necessarily act properly discontinuously. Indeed this is the main source of difficulty in this problem. For getting at least some examples of space-forms in such situations the following construction principle was proposed in [11], namely, construct connected Lie subgroups which act properly and then construct their discrete 
subgroups. In the present case we do this in the following proposition. For simplicity identify $I_{0}\left(S_{1}\right)$ with $P_{1} \times P_{1}$. Let $\phi_{n}: P_{\infty} \rightarrow P_{n}$ denote the canonical covering projection and write $\phi$ for $\phi_{1}$.

Proposition. Up to conjugacy and "switching of factors" the maximal connected Lie subgroups of $I_{0}\left(S_{\infty}\right)$ acting properly on $S_{\infty}$ are of the following types:

(i) $J_{0}=$ the lift to $S_{\infty}$ of the group

$$
\left\{\left(x,\left[\begin{array}{cc}
c & s \\
-s & c
\end{array}\right]^{-}\right) \mid x \in P_{1}, c=\cos \theta, s=\sin \theta\right\} .
$$

(ii) The lifts to $S_{\infty}$ of the groups

$$
\left\{\left(\left[\begin{array}{cc}
1 & a c \\
0 & 1
\end{array}\right]^{-},\left[\begin{array}{cc}
e^{a} & 0 \\
0 & e^{-a}
\end{array}\right]^{-}\right) \mid a \in \mathbf{R}, c, \text { constant } \neq 0\right\}
$$

(iii) The lifts to $S_{\infty}$ of the groups

$$
\left\{\left(\left[\begin{array}{cc}
e^{b t} & 0 \\
0 & e^{-b t}
\end{array}\right]^{-},\left[\begin{array}{cc}
e^{c t} & 0 \\
0 & e^{-c t}
\end{array}\right]\right) \mid t \in \mathbf{R}, b, \text { c real constants, } b \neq c \text { or }-c\right\} .
$$

Proof. The Lie algebra of $I_{0}\left(S_{\infty}\right)$ is $\mathrm{sl}_{2}(\mathbf{R}) \times \mathrm{sl}_{2}(\mathbf{R})$. So the classification of the conjugacy classes of Lie subalgebras and the corresponding connected subgroups is straightforward. The compactness of the isotropy subgroups is a necessary condition for the properness of the action, and it is sufficient, if the action is transitive cf. [11]. In case (i), which with hindsight is the most interesting one, the lift of $\left\{(x, e) \mid x \in P_{1}\right\}$ acts simply transitively on $S_{\infty}$. So $J_{0}$ acts transitively also. The isotropy subgroup at the identity is

$$
\left\{[(x, x)] \mid \phi(x)=\left[\begin{array}{cc}
c & s \\
-s & c
\end{array}\right]^{-}, c=\cos \theta, s=\sin \theta\right\} \approx S^{1} .
$$

So $J_{0}$ acts properly. From the classification of Lie subalgebras referred to above it is easy to see that actually $J_{0}$ is a maximal connected subgroup of $I_{0}\left(S_{\infty}\right)$, not just maximal among the ones which acts properly on $S_{\infty}$. In cases (ii) and (iii), that no strictly larger connected subgroups of $I_{0}\left(S_{\infty}\right)$ can act properly on $S_{\infty}$, will follow from the main lemma of $\S 5$. The groups in (ii) and (iii) act in fact freely. Unfortunately, this does not ensure properness of action in general. That they indeed act properly will also follow from the method of the proof of the main lemma. Since the details are a bit tedious we omit them here.

(2.5). Now consider the group $J=\left\langle J_{0}, i_{u_{0}}\right\rangle$.

Proposition. $J$ acts properly and preserving orientation on $S_{\infty}$. Moreover $J=N_{I\left(S_{\infty}\right)}\left(J_{0}\right)$ and contains $J_{0}$ as a subgroup of index 2 . 
Proof. On the $S_{1}$-level the actions of $\sigma$ and $i_{u_{0}}$ (cf. (2.2)) are as follows. For $x \in S_{1}$ we have $\sigma(x)=x^{-1}, i_{u_{0}}(x)=u_{0} x u_{0}$, and so for $(a, b) \in P_{1} \times P_{1}$,

$$
\begin{aligned}
\sigma \circ(a, b) \circ \sigma^{-1}(x) & =\sigma \circ(a, b)\left(x^{-1}\right)=\sigma\left(a x^{-1} b^{-1}\right)=b x a^{-1}=(b, a)(x), \\
i_{u_{0}} \circ(a, b) \circ i_{u_{0}}^{-1}(x) & =i_{u_{0}} \circ(a, b)\left(u_{0} x u_{0}\right)=i_{u_{0}}\left(a u_{0} x u_{0} b^{-1}\right)=u_{0} a u_{0} x u_{0} b^{-1} u_{0} \\
& =\left(i_{u_{0}}(a), i_{u_{0}}(b)\right)(x) .
\end{aligned}
$$

In other words,

$$
\sigma \circ(a, b) \circ \sigma^{-1}=(b, a), \quad i_{u_{0}} \circ(a, b) \circ i_{u_{0}}^{-1}=\left(i_{u_{0}}(a), i_{u_{0}}(b)\right) .
$$

Lifting to $S_{\infty}$ it follows that $i_{u_{0}}$ normalizes $J_{0}$. Since $i_{u_{0}}^{2}=e$, and $i_{u_{0}}$ preserves orientation of $S_{\infty}$ it follows that $J$ contains $J_{0}$ as a subgroup of index 2 and preserves orientation of $S_{\infty}$. Also $J$ acts transitively on $S_{\infty}$ and the isotropy subgroup at $e$ is isomorphic to $O(2)$, so $J$ acts properly on $S_{\infty}$. Finally let $\alpha \in N_{I\left(S_{\infty}\right)}\left(J_{0}\right)$. Then the image $\bar{\alpha}$ of $\alpha$ in $I\left(S_{1}\right)$ normalizes

$$
\Theta=\left\{\left(x,\left[\begin{array}{cc}
c & s \\
-s & c
\end{array}\right]^{-}\right) \mid x \in P_{1}, c=\cos \theta, s=\sin \theta\right\} .
$$

A computation using the above conjugation formulas shows that $\bar{\alpha} \in\left\langle\Theta, i_{u_{0}}\right\rangle$. Since $J$ is the inverse image of $\left\langle\Theta, i_{u_{0}}\right\rangle$ we must have $\alpha \in J$.

(2.6) Remark. A slightly better assertion is that if $G$ is a group of homeomorphisms of $S_{\infty}$ which acts properly and contains $J_{0}$, then $G=J_{0}$ or $J$. This is proved in [12].

(2.7). We should mention an interesting Lie-group-theoretic perspective on this space-form problem which has partially guided our thinking. It is "dual" to the 3-dimensional group of the 3-dimensional spherical geometry is $O(4)$ whereas that of the linear model in the Lorentz case is $O(2,2)$. Notice that $O(4)$ (resp. $O(2,2)$ ) is locally isomorphic to $O(3) \times O(3)$ (resp. $O(1,2) \times$ $O(1,2))$. The linear model $S^{3}=O(4) / O(3)$ corresponds to $S_{2}=S^{1,2}=$ $O(2,2) / O(1,2)$, whereas the projective model $\mathbf{P}^{3}(\mathbf{R})=O(4) / O(1) \times O(3)$ corresponds to $S_{1}=O(2,2) / O(1) \times O(1,2)$. The novel feature in the Lorentz case arises precisely from the fact that the linear model $S^{1,2}$ has fundamental group $\approx \mathbf{Z}$. The groups of the spherical space-forms up to conjugacy lie in $U(2)$ which is locally isomorphic to $\mathrm{SO}(3) \times \mathrm{SO}(2)$. The group $J_{0}$ described above is akin to $U(2)$ in the spherical case; one analogy is that the "geometry" based on $J_{0}$, like that on $U(2)$, does not allow orientation-reversing isometries.

(2.8). In $\$ 8$ and $\S 9$ we shall put the structures of Lorentz orbifolds on all orientable Seifert orbifolds, coming from $\widehat{\operatorname{SL}(2, R)}$ geometry, by constructing appropriate discrete subgroups of $J$. We shall say a Lorentz orbifold is standard if its "fundamental group" is conjugate to a discrete subgroup of $J$. 
Moreover, we shall also call a discrete subgroup of $I\left(S_{\infty}\right)$ standard if it is conjugate to a subgroup of $J$.

Proposition. Let $\tilde{\Gamma}$ be a subgroup of $\left\langle I_{0}\left(S_{\infty}\right), i_{u_{0}}\right\rangle$. Suppose that $\tilde{\Gamma}$ contains a subgroup $\tilde{\Gamma}_{1}$ of finite index such that $\tilde{\Gamma}_{1}$ is standard then $\tilde{\Gamma}$ is standard.

Proof. Let $\Gamma, \Gamma_{1}$, be the images of $\tilde{\Gamma}, \tilde{\Gamma}_{1}$, resp. in $\left\langle I_{0}\left(S_{1}\right), i_{u_{0}}\right\rangle$. After conjugation, we may assume that $\Gamma_{1}$ is contained in the group

$$
\bar{J}=\left\{\left(x,\left[\begin{array}{cc}
c & s \\
-s & c
\end{array}\right]^{-}\right) \mid x \in P_{1}, c=\cos \theta, s=\sin \theta\right\} \rtimes\left\langle i_{u_{0}}\right\rangle .
$$

It is easy to see that if $g^{n} \in \bar{J}$ then $g \in \bar{J}$. So $\Gamma \subseteq \bar{J}$. Hence $\tilde{\Gamma} \subseteq J$.

(2.9) Corollary. If a Lorentz orbifold $M$ admits a finite orbifold covering which is standard, then either $M$ itself or its double (branched) covering is standard.

$$
\text { 3. Subgroups of } P_{1}=\operatorname{PSL}_{2}(\mathbf{R}) \text {, and } P_{\infty}=\widetilde{\operatorname{PSL}_{2}}(\mathbf{R})
$$

(3.1). In this section we collect together some facts about subgroups of $P_{1}$ and $P_{\infty}$, cf. $\S 2$ for the notation. Let $\phi: P_{\infty} \rightarrow P_{1}$ be the canonical projection.

First, some terminology, not all standard. Consider the three special subgroups of $P_{1}$ :

$$
\begin{gathered}
K=\left\{\left[\begin{array}{cc}
c & s \\
-s & c
\end{array}\right]^{-} \mid c=\cos \theta, s=\sin \theta,-\frac{\pi}{2} \leqslant \theta<\frac{\pi}{2}\right\} \\
A=\left\{\left[\begin{array}{cc}
a & 0 \\
0 & a^{-1}
\end{array}\right]^{-} \mid a \in \mathbf{R}_{>0}\right\}, \quad N=\left\{\left[\begin{array}{cc}
1 & a \\
0 & 1
\end{array}\right]^{-} \mid a \in \mathbf{R}\right\} .
\end{gathered}
$$

Up to conjugacy these are all the 1-parameter subgroups of $P_{1}$, and every element of $P_{1}-\{e\}$ belongs to a unique 1-parameter subgroup. Also $P_{1}=$ $K A N$ is the Iwasawa decomposition. An element $\neq e$ is called elliptic, resp. hyperbolic, resp. parabolic if it is conjugate to an element of $K$, resp. $A$, resp. $N$. Alternately, $x \in P_{1}-\{e\}$ is elliptic, resp. hyperbolic, resp. parabolic iff $Z_{P_{1}}(x)$ is conjugate to $K$, resp. $A$, resp. $N$. Notice that $Z\left(P_{1}\right)=e$. Now the polar decomposition defines a deformation retraction of $P_{1}$ onto $K$, so $\pi_{1}(K, e) \approx \pi_{1}\left(P_{1}, e\right) \approx Z\left(P_{\infty}\right) \approx \mathbf{Z}$, and the standard orientation of $K$ determines a canonical generator, which we call $t$, of $Z\left(P_{\infty}\right)$. A noncentral element $x \in P_{\infty}$ is said to be elliptic, resp. hyperbolic, resp. parabolic if $\phi(x)$ is such. By passing to the Lie algebra it is easy to see that $K, A, N, A N$, are the only proper connected subgroups of $P_{1}$ up to conjugacy. They also happen to be closed.

Proposition. (1) $N_{P_{1}}(K)=K$,

$$
N_{P_{1}}(A)=\left\langle A,\left[\begin{array}{cc}
0 & 1 \\
-1 & 0
\end{array}\right]^{-}\right\rangle \approx \mathbf{R} \rtimes \mathbf{Z}_{2}
$$


where $\mathbf{Z}_{2}$ acts on $\mathbf{R}$ by $x \rightarrow-x$,

(3) $N_{P_{1}}(N)=A N=N_{P_{1}}(A N)$.

(4) $A$ solvable subgroup of $P_{1}$ is conjugate to a subgroup of $K$, or $N_{P_{1}}(A)$, or AN.

(5) $A$ discrete solvable subgroup of $P_{1}$ is either $(a) \approx \mathbf{Z}_{n}$ and is conjugate to a subgroup of $K$, or $(\mathrm{b}) \approx \mathbf{Z}$ and is conjugate to a subgroup of $A$ or of $N$, or (c) $\approx D_{\infty}=$ the infinite dihedral group and is conjugate to a subgroup of $N_{P_{1}}(A)$.

(6) The normalizer of a proper solvable subgroup is solvable.

(7) The commutator of a nonabelian subgroup of $A N$ is contained in $N$. It is either not finitely generated or else it is finitely generated free abelian group of rank at least 2.

The proofs of these statements are not difficult and are omitted.

(3.2) Proposition. (1) $A$ nonsolvable subgroup of $P_{1}$ is either discrete or dense,

(2) A finitely generated dense subgroup of $P_{1}$ contains elliptic elements of infinite order as well as hyperbolic elements.

(3) The normalizer of a discrete nonsolvable subgroup of $P_{1}$ is discrete.

Proof. (1) Let $\Phi$ be a nonsolvable subgroup of $P_{1}, \bar{\Phi}$ its closure. If $\bar{\Phi}_{0}=e$ then $\bar{\Phi}=\Phi$ is discrete. If $\bar{\Phi}_{0}=P_{1}$ then $\Phi$ is dense. If $\bar{\Phi}_{0}$ is conjugate to $K, A$, $N$, or $A N$ then $\Phi$ is contained in $N_{P_{1}}\left(\bar{\Phi}_{0}\right)$ which is solvable. Hence $\bar{\Phi}$ and $\Phi$ would both be solvable.

(2) In $P_{1}$ the set of elliptic, resp. hyperbolic elements is characterized by an open condition $\mid$ trace $\mid<2$, resp. $\mid$ trace $\mid>2$. So a dense subgroup $\Phi$ contains elliptic as well as hyperbolic elements. Passing to a torsion-free subgroup of finite index which must also be clearly dense one sees that $\Phi$ must contain elliptic elements of infinite order as well.

(3) Let $\Phi$ be a discrete nonsolvable subgroup. Then $\Psi=N_{P_{1}}(\Phi)$ is clearly closed. If $\Psi_{0}=e$, then $\Psi$ is discrete. Otherwise, $\operatorname{dim} \Psi>0$ and $\Psi_{0}$ centralizes $\Phi$. But from the knowledge of $Z_{P_{1}}(x)$ it would follow that $\Phi$ is conjugate to a subgroup of $K, A N$ or $N_{P_{1}}(A)$ which would imply that $\Phi$ is solvable.

(3.2.1) Remark. The statements analogous to (3.2) were proved by Nielsen (with quite different proofs), cf. Siegel [33].

(3.3). Now we pass on to subgroups of $P_{\infty}$. Let $\tilde{K}=\phi^{-1}(K)$, and $\tilde{A}=$ $\left(\phi^{-1}(A)\right)_{0}, \quad \tilde{N}=\left(\phi^{-1}(N)\right)_{0}$. An elliptic element of $P_{\infty}$ is conjugate to an element of $\tilde{K}$ whereas a hyperbolic resp. parabolic element of $P_{\infty}$ has the form $t^{n} x$ with $x$ conjugate to an element of $\tilde{A}$, resp. $\tilde{N}$.

Proposition. Let $\Gamma$ be a discrete subgroup of $P_{\infty}$ such that $\Phi=\phi(\Gamma)$ is not discrete. Then either (i) $\Gamma \approx \mathbf{Z}$ and is conjugate to a subgroup of $\tilde{K}$ or (ii) $\Gamma \approx \mathbf{Z}^{2}$ and is conjugate to a subgroup of $\phi^{-1}(A)$ or $\phi^{-1}(N)$. 
Proof. If $\Phi$ is conjugate to a subgroup of $K, A$ or $N$ we are in the situation of (i) or (ii) and are done. Now let $\tilde{\eta} \in \Gamma$ so that $\varphi(\tilde{\eta})=\eta \in \Phi$ is close to $e$ in $\Phi$. There exists $\tilde{\eta}_{1}=\tilde{\eta} h^{k}$ close to $e$ in $\Gamma$. Note $\varphi\left(\tilde{\eta}_{1}\right)=\varphi(\tilde{\eta})$. Observe for $\tilde{\gamma} \in \Gamma$, with $\varphi(\tilde{\gamma})=\gamma,\left[\tilde{\eta}_{1}, \tilde{\gamma}\right]=[\tilde{\eta}, \tilde{\gamma}]$ is close to $e$. Since $\Gamma$ is discrete, $[\tilde{\eta}, \tilde{\gamma}]=e$. Similarly, if we choose $k$ generators $\gamma_{1}, \cdots, \gamma_{k} \in \Phi$ with $\varphi\left(\tilde{\gamma}_{i}\right)=\gamma_{i}, \tilde{\gamma}_{i} \in \Gamma$, we can find an $\tilde{\eta} \in \Gamma$ with $e \neq \varphi(\tilde{\eta})=\eta$ close to $e$ and $\left[\tilde{\eta}, \tilde{\gamma}_{i}\right]=e$ for each $\tilde{\gamma}_{i}$. Therefore $\left\langle\gamma_{i}, \cdots, \gamma_{k}\right\rangle \subseteq C_{\Phi}(\eta) \subset C_{P_{1}}(\eta)$. Since $C_{P_{1}}(\eta)$ is 1-dimensional, and $\Gamma$ is discrete, we must again be in situations (i) and (ii).

(3.5). Before proceeding further it will be helpful to keep the following pictures $^{2}$ of $P_{1}$ and $P_{\infty}$ in mind. First of all $P_{1} \approx S^{1} \times \mathbf{R}^{2}$ may be thought of as an open solid torus. Its fundamental group is carried by $K$. The set $D$ of elements of order 2 forms a single conjugacy class of elliptic elements and so $D \approx P_{1} / K \approx$ an open 2-disk. This disk is closed in $P_{1}$ and cuts $K$ in exactly one point. Hence cutting $P_{1}$ along $D$, taking infinitely many copies of the split $P_{1}$ indexed by $\mathbf{Z}$ and glueing them side by side we obtain $P_{\infty}$, cf. Figure 1 . The set $K-\{e\} \approx \mathbf{R}$ is a set of representatives of conjugacy classes of elliptic elements, and each conjugacy class if $\approx P_{1} / K \approx A N \approx \mathbf{R}^{2}$. So the set $\mathscr{E}$ of elliptic elements in $P_{1}$ is $\approx \mathbf{R}^{3}$. The set

$$
\left\{\left[\begin{array}{cc}
a & 0 \\
0 & a^{-1}
\end{array}\right]^{-} \mid 0<a<1\right\} \approx \mathbf{R}
$$

is a set of representatives of conjugacy classes of hyperbolic elements, and each conjugacy class is $\approx P_{1} / A \approx K N \approx S^{1} \times \mathbf{R}$. So the set $\mathscr{H}$ of hyperbolic elements in $\mathrm{P}_{1}$ is $\approx S^{1} \times \mathbf{R}^{2}$. Finally there are two conjugacy classes of parabolics with representatives

$$
\left[\begin{array}{ll}
1 & 1 \\
0 & 1
\end{array}\right] \text { and }\left[\begin{array}{cc}
1 & -1 \\
0 & 1
\end{array}\right]^{-}
$$

respectively, and each conjugacy class is $\approx P_{1} / N \approx K A \approx S^{1} \times \mathbf{R}$. So the set $\mathscr{P}$ of parabolic elements in $P_{1}$ consists of two disjoint copies of $S^{1} \times \mathbf{R}$. Thus $P_{1}$ is a disjoint union of $\{e\}, \mathscr{E}, \mathscr{H}$, and $\mathscr{P}$. The relative position of these sets is pictured in the Figure 1. The set $D$ of elements of order 2 may be thought of as a "meridian disk". One component of the "boundary" of each of the "cylinders" (which together make up $\mathscr{P}$ ) is "pinched at $e$ ", whereas the other component coincides with the boundary of $D$. The 2-disks which constitute the conjugacy classes of elliptics parametrized by $-\pi / 2 \leqslant \theta<\pi / 2$ go in a snakelike fashion inside the cylinders of parabolics (which of the two cylinders is

\footnotetext{
${ }^{2}$ This discussion is inspired by $\S 2$ of [18].
} 
decided by $\theta>0$ or $\theta<0$ ) and also have their boundaries coinciding with the boundary of $D$. The reader may verify these statements computationally by working out the behavior of polar decompositions of elements in a single conjugacy class.
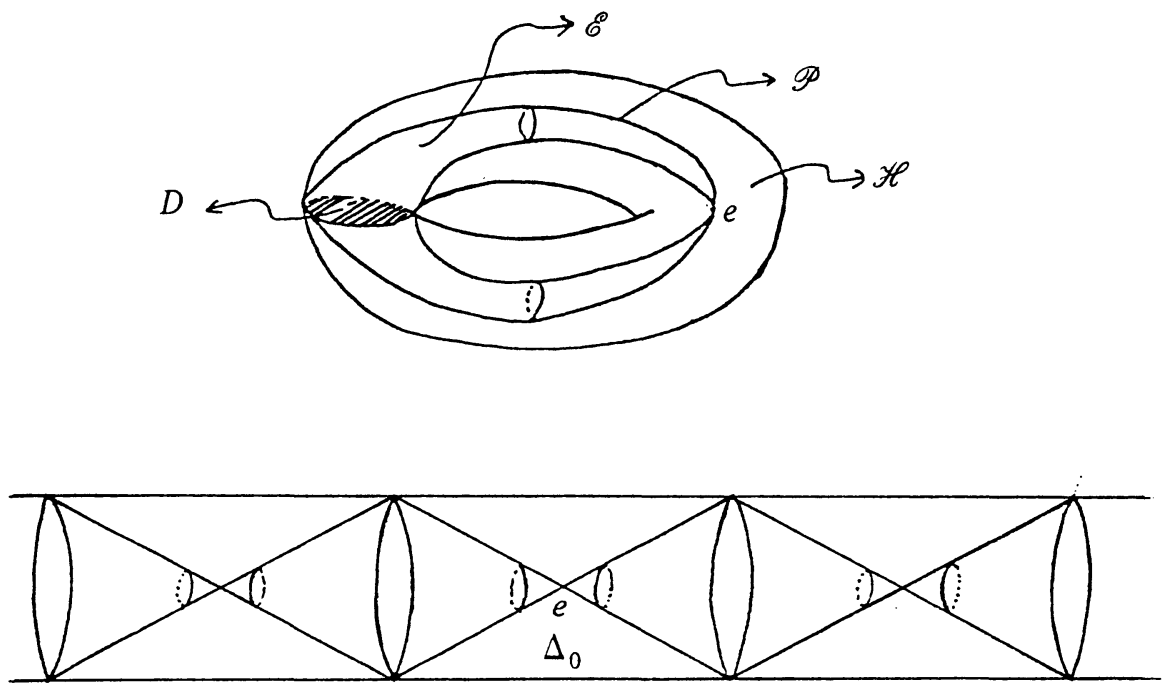

FIGURE 1

The Lorentz geometry is naturally "built in" for $\operatorname{PSL}_{2}(\mathbf{R})$. The set $\mathscr{P} \cup\{e\}$ is the light-cone at $e$. The time-like (resp. space-like) geodesics through $e$ are precisely the subgroups conjugate to $K$ (resp. $A$ ). The geodesics and the light-cones at other points are obtained by either left- or right-translation.

The polar decomposition induces $\rho: P_{1} \rightarrow \mathrm{SO}(2) / \pm 1$ given by

$$
\rho\left(\left[\begin{array}{ll}
a & b \\
c & d
\end{array}\right]\right)^{-}=\theta \quad \text { if } \theta=\arctan ((b-c) /(a+d)),
$$

cf. $[M n]_{1}$. Lifting to $P_{\infty}$ we have a "polar decomposition" inducing $\rho_{\infty}$ : $P_{\infty} \rightarrow \mathbf{R}$. Then $\Delta_{0}=\rho_{\infty}^{-1}\{(-\pi / 2, \pi / 2]\}$ is clearly a fundamental domain for the action of $Z\left(P_{\infty}\right)=\langle t\rangle$.

The image of the exponential map from $\mathrm{sl}_{2}(\mathbf{R})$ to $P_{1}$ is clearly surjective, and is never surjective on $P_{n}$ for $n \geqslant 2$. On $P_{\infty}$ the image of the exponential map is precisely $\Delta_{0} \cup$ \{the elliptic elements $\}$. An element in the image of the exponential map in $P_{\infty}$ is said to be of exponential type.

Proposition. A nonabelian free subgroup of $P_{\infty}$ contains a hyperbolic element of exponential type. 
Proof. Let $\Gamma$ be a nonabelian free subgroup of $P_{\infty}$ and $\Phi=\Phi(\Gamma)$ its image in $P_{1}$. Clearly, $\phi: \Gamma \rightarrow \Phi$ is an isomorphism. For $x \in \Phi$ let $\tilde{x}$ be the unique element in $\Gamma$, and $\tilde{x}_{0}$ the unique element in $\Delta_{0}$ such that $\phi(\tilde{x})=\phi\left(\tilde{x}_{0}\right)$ $=x$. Clearly $\tilde{x}=t^{n} \tilde{x}_{0}$ for a suitable $n \in \mathbf{Z}$.

Next it is elementary to see, using (3.1) and (3.2), that $\Phi$ contains a nonabelian free subgroup generated by two hyperbolic elements. So passing to a subgroup and by conjugation we may take $\Phi=\langle x, y\rangle$ where

$$
\begin{gathered}
x=\left[\begin{array}{cc}
\lambda & 0 \\
0 & \lambda^{-1}
\end{array}\right]^{-}, \quad \lambda>1, \\
y=\left[\begin{array}{ll}
a & b \\
c & d
\end{array}\right]^{-}, \quad a+d>2 .
\end{gathered}
$$

Since $\Gamma$ is nonabelian and free we see that $b c \neq 0$. We claim that $\Gamma$ must contain an element

$$
z=\left[\begin{array}{cc}
l & m \\
n & p
\end{array}\right] \text { with } m n<0
$$

Indeed, if $b c<0$ we may take $z=y$. Otherwise suppose $b c>0$. Then $a d=1$ $+b c>0$. A computation shows that, for $z=y^{-1} x y$ the product of the off-diagonal elements is $-a b c d\left(\lambda-\lambda^{-1}\right)^{2}<0$. So changing notation we may assume $\Phi=\langle x, y\rangle$ as above with $b c<0$. Now consider

$$
A=\left\{x_{r}=\left[\begin{array}{cc}
r & 0 \\
0 & r^{-1}
\end{array}\right]^{-} \mid r>0\right\} .
$$

A computation shows that $z_{r}=x_{r} y x_{r}^{-1} y^{-1}$ has $\mid$ trace $\mid=2-b c\left(r-r^{-1}\right)^{2}>2$ since $b c<0$. So $r \rightarrow z_{r}$ is a curve starting at $e$ and consists of hyperbolic elements of exponential type.

Now let $\tilde{x}=t^{n} \tilde{x}_{0}, \tilde{y}=t^{m} \tilde{y}_{0}$, be elements of $\Gamma$ lying above $x, y$, resp. The curve $r \rightarrow t^{n} \tilde{x}_{r 0}=\tilde{x}_{r}$, say, lies over $r \rightarrow x_{r}$ and contains $\tilde{x}$. So the curve $r \rightarrow \tilde{x}_{r} \tilde{y} \tilde{x}_{r}^{-1} \tilde{y}^{-1}=\tilde{z}_{r}$ lies over $r \rightarrow z_{r}$ and starts at $e$. Conclusion: $\tilde{x} \tilde{y} \tilde{x}^{-1} \tilde{y}^{-1} \in \Gamma$ is a hyperbolic element of exponential type.

\section{Subgroups of $I_{0}\left(S_{\infty}\right)$, the level of a subgroup}

(4.1). Throughout this section $\tilde{\Gamma}$ denotes a subgroup of $I_{0}\left(S_{\infty}\right), \phi: I_{0}\left(S_{\infty}\right)$ $\rightarrow I_{0}\left(S_{1}\right)$ the canonical projection, and $\phi(\tilde{\Gamma})=\Gamma$. We identify $I_{0}\left(S_{1}\right)$ with $P_{1} \times P_{1}$ and consider its projections $p_{1}$ and $p_{2}$ in the first and second factor resp. Write $\Gamma_{i}=p_{i}(\Gamma), \Psi_{1}=\left.\operatorname{ker} p_{2}\right|_{\Gamma}$, and $\Psi_{2}=\left.\operatorname{ker} p_{1}\right|_{\Gamma}$. Also write $Z=$ $Z\left(I_{0}\left(S_{\infty}\right)\right)$. We have $Z \approx \mathbf{Z}$. Fix its generator $h=[(t, e)]$. Define $\tilde{\Gamma}$ to be of level $n$ if $n$ is the least positive integer (possibly infinite) such that $\tilde{\Gamma}$ is the 
complete inverse image of a subgroup of $I_{0}\left(S_{n}\right)$ i.e. iff $\tilde{\Gamma} \cap Z=\left\langle h^{n}\right\rangle$. It is clear that if $\tilde{\Gamma}$ has finite level then it is discrete (resp. acts properly discontinuously on $S_{\infty}$ ) iff $\Gamma$ is discrete (resp. acts properly discontinuously on $S_{1}$ ). We shall study properness of actions in the next section. Here we investigate the consequences of discreteness of $\tilde{\Gamma}$ of infinite level. For characterizing these groups notice that if $\tilde{\Gamma}$ is of infinite level $\phi: \tilde{\Gamma} \rightarrow \Gamma$ is an isomorphism. Since $\Gamma, \Gamma_{1}, \Gamma_{2}$ are all linear groups, under the hypothesis of finite generation, they contain torsion-free subgroups of finite index. Therefore, we lose no generality in assuming that $\tilde{\Gamma} \approx \Gamma, \Gamma_{1}, \Gamma_{2}$ are all torsion free.

(4.2) Theorem. Let $\tilde{\Gamma}$ be a finitely generated discrete subgroup of $I_{0}\left(S_{\infty}\right)$ of infinite level such that $\tilde{\Gamma} \approx \Gamma, \Gamma_{1}, \Gamma_{2}$ are all torsion free. Then either (1) $\Gamma$ is discrete, or (2) $\Gamma$ is abelian but not discrete with the following possibilities:

(i) $\Gamma=\langle(x, y)\rangle \approx \mathbf{Z}, x$ and $y$ elliptic and one of them is of infinite order, or;

(ii) $\Gamma \approx \mathbf{Z}^{2}$, contained in $B \times$ e or $e \times B$ where $B$ is conjugate to $A$ or $N$-or else contained in $B \times C$ where one of $B$ and $C$ is conjugate to $K$ and the other conjugate to $A$ or $N$ :

(iii) $\Gamma \approx \mathbf{Z}^{3}$ contained in $B \times C$ where both $B$ and $C$ are conjugate to $A$ or $N$; or (3) $\Gamma$ is nonabelian but not discrete and is contained in $P_{1} \times B$ (up to switching the factors) where $B$ is conjugate to $K$ or $A$ or $N$. In this case $\Gamma$ has central elements of the form $(e, y), y \in B$ arbitrarily close to $e$. Moreover, there exists a discrete nonabelian free subgroup $\Phi$ of $P_{1} \times e$ and a representation $\rho$ : $\Phi \rightarrow B$ such that $\Gamma=\left\langle\Phi_{\rho}, \Psi_{2}\right\rangle \approx \Phi_{\rho} \times \Psi_{2}$ where

$$
\Phi_{\rho}=\{(x, \rho(x)) \mid x \in \Phi\} \quad \text { and } \quad \Psi_{2}=\operatorname{ker} p_{1}
$$

Proof. Suppose $\Gamma$ is not discrete. If $\Gamma$ is abelian then by looking at the generators and their possible centralizers it is not difficult to see that we have the only possibilities listed in (2). Now suppose $\Gamma$ is nonabelian.

We first claim that it has a nontrivial center. Let $\tilde{\gamma}, \tilde{\eta} \in \tilde{\Gamma}, \phi(\tilde{\gamma})=\gamma$, $\phi(\tilde{\eta})=\eta$ such that $\eta$ is close to $e$. Then there exists $\tilde{\eta}_{1}$ close to $e$ such that $\tilde{\eta}_{1}=h^{k} \tilde{\eta}$ for some $k \in \mathbf{Z}$. But then $[\tilde{\gamma}, \tilde{\eta}]=\left[\tilde{\gamma}, \tilde{\eta}_{1}\right]$ is close to $e$. Since $\tilde{\Gamma}$ is finitely generated and discrete we see that there exists $\tilde{\eta} \neq e$ such that $[\tilde{\gamma} ; \tilde{\eta}]=e$ for all $\tilde{\gamma} \in \tilde{\Gamma}$.

Choose a nontrivial element $\eta=(x, y)$ in $Z(\Gamma)$. If $x \neq e \neq y$ then $\Gamma \subseteq$ $Z_{P_{1} \times P_{1}}(\eta)$ would be abelian. So up to switching factors suppose that $x=e \neq y$. If $B=Z_{P_{1}}(y)$ we see that $\Gamma \subseteq P_{1} \times B$ with $B$ conjugate to $K, A$, or $N$. The above argument shows that $\Psi_{2}=\operatorname{ker} p_{1}$ is not discrete. Also $\Psi_{1}=\operatorname{ker} p_{2}$ must be discrete for otherwise $\Psi_{1} \times \Psi_{2} \subseteq \Gamma$ would contain elements of the form $(x, y), x \neq e \neq y$ arbitrarily close to $e$ and as above $\Gamma$ would be abelian. Now 
$\Gamma_{1}$ normalizes $\Psi_{1}$. If $\Psi_{1}=e, \Gamma \approx p_{2}(\Gamma) \subseteq B$ would be abelian. If $\Psi_{1} \approx \mathbf{Z}$, $\Gamma \subseteq N_{P_{1}}\left(\Psi_{1}\right) \times B$ would again be abelian-use (3.1), part (7). Hence $\Psi_{1}$ is a nonsolvable discrete subgroup of $P_{1}$, so is $\Gamma_{1}$ by (3.2), part (3). Write $\Phi=\Gamma_{1}$. We know that $\Phi \approx$ a finitely generated nonabelian free group or $\approx \pi_{1}$ (a closed surface of genus $\geqslant 2$ ). In the first case let $\left\{x_{1}, \cdots, x_{r}\right\}$ be a set of free generators whereas in the second case let $\left\{a_{i}, b_{i}\right\}, i=1,2, \cdots, g$, be a set of generators so that $\prod_{i=1}^{g}\left(a_{i}, b_{i}\right)=e$. By construction, in the first case there exist $\left\{y_{1}, \cdots, y_{r}\right\}$ such that $\left(x_{i}, y_{i}\right) \in \Gamma$ whereas in the second case there exist $\left\{a_{i}^{\prime}, b_{i}^{\prime}\right\}$ such that $\left(a_{i}, a_{i}^{\prime}\right),\left(b_{i}, b_{i}^{\prime}\right)$ are in $\Gamma$. Since $B$ is abelian we see that in either case $\rho\left(x_{i}\right)=y_{i}$ resp. $\rho\left(a_{i}\right)=a_{i}^{\prime}, \rho\left(b_{i}\right)=b_{i}^{\prime}$ defines a representation $\rho$ : $\Phi \rightarrow \beta$. Then writing $\Phi_{\rho}=\left\{(x, \rho(x)) \mid x \in P_{1}\right\}$ we see that $\Phi_{\rho} \cap \Psi_{2}=e$ and $\Gamma=\left\langle\Phi_{\rho}, \Psi_{2}\right\rangle \approx \Phi_{\rho} \times \Psi_{2}$.

The final claim is that $\Phi$ cannot be isomorphic to $\pi_{1}$ (closed surface of genus $\geqslant 2$ ). For if so, let $a_{i}, b_{i}$ be as above and let $\tilde{a}_{i}, \tilde{b}_{i} \in P_{\infty} \times e \rightarrow I_{0}\left(S_{\infty}\right)$ and $a_{i}^{\prime}, b_{i}^{\prime} \in P_{\infty} \rightarrow I_{0}\left(S_{\infty}\right)$ be some lifts of $a_{i}, b_{i}$ and $\rho\left(a_{i}\right), \rho\left(b_{i}\right)$ resp. so that $\left[\left(\tilde{a}_{i}, a_{i}^{\prime}\right)\right],\left[\left(\tilde{b}_{i}, b_{i}^{\prime}\right)\right]$ are in $\tilde{\Gamma}$. Then

$$
\begin{aligned}
\prod_{i=1}^{g}\left[\left[\left(\tilde{a}_{i}, a_{i}^{\prime}\right)\right],\left[\left(\tilde{b}_{i}, b_{i}^{\prime}\right)\right]\right] & =\left[\left[\prod_{i=1}^{g}\left(\tilde{a}_{i}, \tilde{b}_{i}\right), \prod_{i=1}^{g}\left(a_{i}^{\prime}, b_{i}^{\prime}\right)\right]\right] \\
& =\left[\left(t^{2 g-2}, e\right)\right]=h^{2 g-2}
\end{aligned}
$$

by [28, Theorem 1]. So $\Gamma \cap Z \neq e$ which contradicts the hypothesis that $\tilde{\Gamma}$ is of infinite level.

\section{Properly discontinuous subgroups of $I_{0}\left(S_{1}\right)$}

(5.1). As in earlier sections we identify $I_{0}\left(S_{1}\right)$ with $P_{1} \times P_{1}$ which acts on $S_{1}$ via $(a, b): x \rightarrow a x b^{-1}$. The following lemma is crucial.

Main lemma. Let $\Gamma$ be a subgroup of $I_{0}\left(S_{1}\right)$ which acts properly discontinuously on $S_{1}$. Then $\Gamma$ does not contain a subgroup $\approx \mathbf{Z}^{2}$.

Proof. Suppose that the lemma is false. Then we may take $\Gamma \approx \mathbf{Z}^{2}$. Certainly $\Gamma$ is a discrete subgroup so from (3.1) part (5) it is not entirely contained in $P_{1} \times e$ or $e \times P_{1}$. (Alternately, this is also well known from the plane hyperbolic geometry.) So there exists $(x, y) \in \Gamma, x \neq e \neq y$, hence $\Gamma \subseteq Z_{P_{1} \times P_{1}}((x, y))=Z_{P_{1}}(x) \times Z_{P_{1}}(y)$.

Case 1. Assume that $x$ or $y$, say $x$, is elliptic. So $Z_{P_{1} \times P_{1}}((x, y))$ is conjugate to $K \times B$ where $B=K, A$, or $N$. In any case $K \times B \approx S^{1} \times S^{1}$ or $S^{1} \times \mathbf{R}$ which cannot contain a discrete subgroup $\approx \mathbf{Z}^{2}$-a contradiction. 
Case 2. Assume that $x$ and $y$ are hyperbolic. Then $G=Z_{P_{1} \times P_{1}}((x, y))$ is conjugate to $A \times A \approx \mathbf{R}^{2}$ and contains $\Gamma \approx \mathbf{Z}^{2}$ as a discrete, hence cocompact subgroup. Since $\Gamma$ acts properly discontinuously, $G$ must act properly cf. [11, §1]. In particular, then, $\Gamma^{\prime}=\langle(x, e),(e, y)\rangle$ must act properly discontinuously. Modulo conjugation, taking inverses, and switching of factors we may take

$$
x=\left[\begin{array}{cc}
a & 0 \\
0 & a^{-1}
\end{array}\right]^{-}, \quad y=\left[\begin{array}{cc}
b & 0 \\
0 & b^{-1}
\end{array}\right]^{-}, \quad 1<a \leqslant b .
$$

So

$$
\left(x^{m}, y^{n}\right) e=\left[\begin{array}{cc}
a^{m} b^{-n} & 0 \\
0 & a^{m} b^{-n}
\end{array}\right]^{-} .
$$

But for infinitely many choices of $m, n$ this element comes close to $e$-take rational approximations $m / n$ to $-\ln b / \ln a$ where $m, n$ are not necessarily coprime. Then either the isotropy subgroup $\Gamma_{e}^{\prime}$ is infinite or the orbit $\Gamma^{\prime}(e)$ is not discrete which contradicts proper discontinuity of $\Gamma^{\prime}$.

Case 3. Assume that $x$ and $y$ are parabolic. Then modulo conjugation in $I\left(S_{1}\right)$ we may take

$$
x=\left[\begin{array}{ll}
1 & 1 \\
0 & 1
\end{array}\right]^{-}=y \text {. }
$$

But then $(x, y)$ has infinite order and fixes $e$ which contradicts proper discontinuity of $\langle(x, y)\rangle$.

Case 4. Assume that, up to switching of factors, $x$ is hyperbolic and $y$ is parabolic. Then $Z_{P_{1} \times P_{1}}((x, y))$ is conjugate to $A \times N \approx \mathbf{R} \times \mathbf{R}$, and as in Case 2 the group $\Gamma^{\prime}=\langle(x, e),(e, y)\rangle$ acts properly discontinuously. Modulo conjugation in $I\left(S_{1}\right)$ we may take

$$
x=\left[\begin{array}{cc}
\lambda & 0 \\
0 & \lambda^{-1}
\end{array}\right]^{-}, \quad y=\left[\begin{array}{ll}
1 & 1 \\
0 & 1
\end{array}\right]^{-}, \quad 1<\lambda .
$$

Let

$$
C=\left\{\left[\begin{array}{ll}
a & b \\
c & d
\end{array}\right]^{-}|| a|+| b|+| c|+| d \mid \leqslant 4\right\}
$$

which is a compact subset of $S_{1}$. Fix a positive integer $m$ and $n=\left[\lambda^{m}\right]$, so $n=\lambda^{m}-\varepsilon, 0 \leqslant \varepsilon<1$. Then

$$
\alpha=\left[\begin{array}{cc}
\lambda^{-m} & -1 \\
1-\lambda^{-m} & 1
\end{array}\right]^{-} \in C
$$

and also

$$
\left(x^{m}, y^{-n}\right) \alpha=\left[\begin{array}{cc}
1 & -\varepsilon \\
\lambda^{-m}-\lambda^{-2 m} & 1-\varepsilon \lambda^{-m}+\varepsilon \lambda^{-2 m}
\end{array}\right]^{-} \in C .
$$


Thus for infinitely many pairs $(m, n)$

$$
\left\{\left(x^{m}, y^{-n}\right) C\right\} \cap C \neq \varnothing
$$

contradicting the proper discontinuity of $\Gamma^{\prime}$ and finishing the proof.

(5.2) Theorem. Let $\Gamma$ be torsion-free subgroup of $I_{0}\left(S_{1}\right)$ acting properly discontinuously on $S_{1}$. Then, up to switching of factors, $\Gamma=\Phi_{\rho}$ where $\Phi$ is a discrete subgroup of $P_{1}, \rho: \Phi \rightarrow P_{1}$ a representation, and $\Phi_{\rho}=\{(x, \rho(x)) \mid x \in$ $\Phi\}$.

Proof. Let $p_{j}: P_{1} \times P_{1} \rightarrow P_{1}, j=1,2$, be the projections onto the first and second factors. If $\operatorname{ker} p_{j \mid \Gamma} \neq e$ for both $j=1,2$, there exist elements $(x, e)$, $(e, y) \quad x \neq e \neq y$ in $\Gamma$ generating $\mathbf{Z}^{2}$ which contradicts (5.1). So up to switching factors, suppose $p_{1 \mid \Gamma}$ is injective. Set $\Phi=p_{1}(\Gamma)$ and $\rho=\left.p_{2}{ }^{\circ} p_{1}^{-1}\right|_{\Phi}$. So $\Gamma=\Phi_{\rho}$. We still need to show that $\Phi$ is discrete.

Case 1. Assume that $\Psi_{1}=\operatorname{ker} p_{2 \mid \Gamma} \neq e$. Then $\Psi_{1}=\Gamma \cap \Phi$ is a discrete normal subgroup of $\Phi$. If $\Psi_{1}$ is not solvable then $\Phi$ is discrete, cf. (3.2) part (3). If $\Psi_{1}$ is solvable, it is $\approx \mathbf{Z}$ and $\Phi$ is solvable cf. (3.1) parts (5), (6). An easy analysis shows that if $\Phi$ is not discrete then $\Phi$ contains a subgroup $\approx \mathbf{Z}^{2}$. But then $\Gamma \approx \Phi$ would contain a subgroup $\approx \mathbf{Z}^{2}$ contradicting (5.1).

Case 2. Assume that $\operatorname{ker} p_{2 \mid \Gamma}=e$. If $\Phi$ is nondiscrete but $\rho(\Phi)=p_{2}(\Gamma)$ is discrete we can simply switch the factors. So assume, if possible, that both $\Phi$ and $\rho(\Phi)$ are nondiscrete. If $\Gamma \approx \mathbf{Z}$, let $(x, y)$ be its generator. Since $\Phi$ and $\rho(\Phi)$ are nondiscrete both $x$ and $y$ must be elliptic of infinite order. But then $\Gamma$ would not be discrete. So $\Gamma \neq \mathbf{Z}$. Then $\Gamma$ is nonsolvable, for otherwise $\Phi$ would be a nondiscrete solvable subgroup of $P_{1}$ and so $\Gamma \approx \Phi$ would contain a subgroup $\approx \mathbf{Z}^{2}$ contradicting (5.1). So $\Gamma \approx \Phi \approx \rho(\Phi)$ are nonsolvable. Then $\Phi$ and $\rho(\Phi)$ being nondiscrete would be dense in $P_{1} \times e$ and $e \times P_{1}$ resp., and would contain elliptic elements of infinite order by (3.2) i.e. there exist $(x, v)$, $(u, y)$ in $\Gamma$ with $u$ and $v$ elliptic. Now $x, y$, must be nonelliptic, otherwise $\Gamma$ would not be discrete. Consider $B=$ the closure of $\langle(e, v),(u, e)\rangle$. Since $B$ is compact $\Gamma B$ is a proper family cf. $[11, \S 1]$ and contains the subgroup $H=\langle(x, e),(e, y)\rangle$. Moreover, since $x, y$ are nonelliptic $H$ is a closed subset of $\Gamma B$ and would act properly. But then $H \approx \mathbf{Z}^{2}$ which again contradicts (5.1).

\section{Solvable fundamental groups}

(6.1). We shall use the notations introduced in (4.1).

Theorem. Let $M$ be a Lorentz space-form with $\pi_{1}(M)=\tilde{\Gamma}$, which, realized as a deck-transformation group is contained in $I_{0}\left(S_{\infty}\right)$ and solvable. Then $\tilde{\Gamma} \approx \mathbf{Z}$, $\mathbf{Z}^{2}$, or $\pi_{1}$ (Klein bottle). Also, volume $(M)$ is infinite, and, in particular, $M$ is not compact. 
Proof. Since $\tilde{\Gamma}$ acts freely and properly discontinuously on $S_{\infty} \approx \mathbf{R}^{3}$ it is torsion-free, discrete, and of cohomological dimension $\leqslant 3$. If $\tilde{\Gamma}$ has finite level then $\Gamma=\phi(\tilde{\Gamma})$ acts properly discontinuously on $S_{1}$, and so it is a discrete solvable subgroup of $I_{0}\left(S_{1}\right)=P_{1} \times P_{1}$. By (5.1), $\Gamma$ does not contain a subgroup $\approx \mathbf{Z}^{2}$. By using (3.1) part (5), then $\Gamma \approx \mathbf{Z}_{n}, \mathbf{Z}, \mathbf{Z}_{n} \oplus \mathbf{Z}, D_{\infty}$, or $\mathbf{Z}_{n} \oplus D_{\infty}$ and $\tilde{\Gamma} \approx \mathbf{Z}, \mathbf{Z}^{2}$, or $\pi_{1}$ (Klein bottle). So suppose that $\tilde{\Gamma}$ has infinite level. Then $\phi: \tilde{\Gamma} \rightarrow \Gamma$ is an isomorphism. If $\Gamma$ is discrete (and torsion-free) one sees that $\tilde{\Gamma} \approx \Gamma \approx \mathbf{Z}$ or $\mathbf{Z}^{2}$. So suppose $\Gamma$ is not discrete. First we claim that $\Gamma$ cannot contain a subgroup $\approx \mathbf{Z}^{3}$. For otherwise, by the consideration of cohomological dimension and passing to a subgroup of finite index we may take $\tilde{\Gamma} \approx \mathbf{Z}^{3}$ and $M$ is compact. By (4.2), Case 2 (iii), this happens only when $\Gamma \subseteq B \times C$ $\approx \mathbf{R}^{2}$, so $\tilde{\Gamma} \subseteq \phi^{-1}(B \times C) \approx \mathbf{R}^{2} \times \mathbf{Z}$ and $\tilde{\Gamma}$ must be cocompact in $\phi^{-1}(B \times C)$. Since $\tilde{\Gamma}$ acts properly so does $\phi^{-1}(B \times C)$ cf. [11, §1]. But then $\phi^{-1}(B \times C) / Z$ $=B \times C$ would also act properly contradicting (5.1). Hence $\Gamma$ does not contain a subgroup $\approx \mathbf{Z}^{3}$.

Now we consider two cases.

Case 1. Assume $\Gamma$ is abelian. Then from the above argument it follows that any finitely generated subgroup of $\Gamma$ is $\approx \mathbf{Z}$ or $\mathbf{Z}^{2}$. The part (2)(i)(ii) of (4.2) lists these possibilities and again the consideration of possible centralizers of elements in $P_{1} \times P_{1}$ and their inverse images in $I_{0}\left(S_{\infty}\right)$ shows that $\tilde{\Gamma}$ must be finitely generated and $\approx \mathbf{Z}$ or $\mathbf{Z}^{2}$.

Case 2. Assume $\Gamma$ to be nonabelian. Then the beginning of the proof of (4.2), Case 3 shows that $Z(\Gamma) \neq e$ and in fact $\Gamma$ cannot be solvable. This contradiction finishes the proof.

(6.2) Remark. The $\mathbf{Z}$ 's or $\mathbf{Z}^{2}$ 's acting properly discontinuously on $S_{\infty}$ can be easily classified from the above proof, cf. also (2.4). Notice that the only subgroups $\approx \mathbf{R}^{2}$ of $I_{0}\left(S_{\infty}\right)$ which act properly are up to conjugacy, contained in $J_{0}$ and are conjugate to $\tilde{A} \times \tilde{K}$ or $\tilde{N} \times \tilde{K}$ (cf. (3.3)).

\section{Finiteness of level}

(7.1). Throughout this section we use the notations of (4.1), namely, $\tilde{\Gamma}$ is a subgroup of $I_{0}\left(S_{\infty}\right), Z=Z\left(I_{0}\left(S_{\infty}\right)\right)=\langle h\rangle, \phi: I_{0}\left(S_{\circ}\right) \rightarrow I_{0}\left(S_{1}\right)=P_{1} \times P_{1}$, the canonical projection and $p_{i}: P_{1} \times P_{1} \rightarrow P_{1}, i=1,2$, the projections into the first and second factor resp., $\phi(\tilde{\Gamma})=\Gamma, p_{i}(\Gamma)=\Gamma_{i}, \Psi_{1}=\left.\operatorname{ker} p_{2}\right|_{\Gamma}, \Psi_{2}=$ $\left.\operatorname{ker} p_{1}\right|_{\Gamma}$. Moreover as explained in (4.1), by passing to subgroups of finite index, we may assume that for finitely generated $\Gamma$, all the groups $\tilde{\Gamma}, \Gamma, \Gamma_{i}$ are torsion-free and finitely generated. The Theorem (7.2) below shows that a compact space-form has finite level, whereas (7.1) partially describes the 
structure of space-forms which are not necessarily of finite level.

Theorem. Let $M=\tilde{\Gamma} \backslash S_{\infty}$ be a space-form with $\tilde{\Gamma} \approx \pi_{1}(M)$ finitely generated and contained in $I_{0}\left(S_{\infty}\right)$. Assume that $\tilde{\Gamma}, \Gamma, \Gamma_{i}$ are torsion-free. Then one of the following possibilities occur.

(1) $M$ has finite level,

(2) $\tilde{\Gamma} \approx \mathbf{Z}$ or $\mathbf{Z}^{2}$,

(3) There exists a subgroup $\Phi$ of $P_{1}$ and a representation $\rho: \Phi \rightarrow P_{1}$ such that, up to switching of factors, and writing $\Phi_{\rho}=\{(x, \rho(x)) \mid x \in \Phi\}$ we have either

(i) $\tilde{\Gamma}=\left\langle\Phi, \Psi_{2}\right\rangle \approx \Phi_{\rho} \times \Psi_{2}$ where $\Psi_{2} \approx \mathbf{Z}$ and $\Phi \approx \Gamma_{1}$ is a discrete nonabelian free subgroup of $P_{1}$; moreover $\Gamma$ is not discrete iff $\Psi_{2}$ is contained in a subgroup conjugate to $K$, or

(ii) $\tilde{\Gamma}=\Phi_{\rho}$; moreover if $\rho$ is not an isomorphism then $\Phi$ is a discrete nonabelian free subgroup of $P_{1}$.

Proof. Suppose the possibilities (1) and (2) do not occur. So in particular $\phi: \tilde{\Gamma} \rightarrow \Gamma$ is an isomorphism. First consider the case when there exists $\tilde{z} \in Z_{I_{0}\left(S_{\infty}\right)}(\tilde{\Gamma})-Z$. (By (4.2), this is necessarily the case if $\Gamma$ is not discrete.) Let $\phi(\tilde{z})=(x, y)$. We cannot have $x \neq e \neq y$, for otherwise $\Gamma \subseteq$ $Z_{P_{1} \times P_{1}}((x, y))$ would be abelian and hence $\approx \mathbf{Z}$ or $\mathbf{Z}^{2}$ by (6.1). So, up to switching of factors, assume that $x=e \neq y$. Then, $\Gamma \subseteq P_{1} \times B$ where $B=$ $Z_{P_{1}}(y)$ is a 1-parameter group.

Subcase $\alpha$. $\Gamma$ not discrete. Then by (4.2), $\Gamma=\left\langle\Phi_{\rho}, \Psi_{2}\right\rangle$ with $\Phi=a$ discrete nonabelian free subgroup of $P_{1}$, and $\rho: \Phi \rightarrow B$ a suitable representation. Also $\Gamma_{2}$, and hence $\Psi_{2}$, are finitely generated abelian groups. If $\Psi_{2}$ contains a subgroup $\approx \mathbf{Z}^{2}$, then $\tilde{\Gamma} \approx \Gamma$ would contain a subgroup $\theta \approx \Phi \times \mathbf{Z}^{2}$. The latter has cohomological dimension 3 hence $\theta \backslash S_{\infty}$ would be compact. But then $\theta$ would be a Poincaré duality group, but $\Phi \times \mathbf{Z}^{2}$ is not. Hence $\Psi_{2} \approx \mathbf{Z}$. Moreover, if $B$ is conjugate to $A$ or $N$, clearly $\Gamma$ would be discrete. Since we are assuming $\Gamma$ to be nondiscrete, $B$ must be conjugate to $K$.

Subcase $\beta$. $\quad \Gamma$ discrete. So $\Psi_{1}, \Psi_{2}$ are discrete. Also $\Gamma_{2}$ is abelian, so $\Psi_{2} \approx \mathrm{Z}$ and $B$ is conjugate to $A$ or $N$. If $\Psi_{1}$ is abelian it is clear that $\Gamma$ would be abelian, hence $\approx \mathbf{Z}$ or $\mathbf{Z}^{2}$ by (6.1). So $\Psi_{1}$ is nonabelian, discrete and torsion free, hence nonsolvable, and $\Gamma_{1}$ which normalizes $\Psi_{1}$ is a discrete subgroup of $P_{1}$, cf. (3.1), (3.2). Write $\Gamma_{1}=\Phi$. Exactly as in the last part of (4.2) we see that $\Gamma=\left\langle\Phi_{\rho}, \Psi_{2}\right\rangle \approx \Phi \times \Psi_{2}$ for a suitable representation $\rho: \Phi \rightarrow B$. Moreover as in (4.2) we see that $\Phi$ must be isomorphic to a nonabelian free group.

Now consider the case when no such element $\tilde{z}$ exists. Then, since by hypothesis $\tilde{\Gamma}$ has infinite level, $\tilde{\Gamma} \approx \Gamma$ has trivial center, and by (4.2), $\Gamma$ is necessarily discrete. We claim that $p_{1}$ and $p_{2}$ cannot both fail to be isomorphisms. For otherwise $\Psi_{1} \neq e \neq \Psi_{2}$. If either of $\Psi_{1}$ or $\Psi_{2}$ is abelian, clearly 
$Z(\Gamma) \neq e$. So $\Psi_{1}, \Psi_{2}$ are both nonabelian and discrete, hence contain nonabelian free subgroups consisting (necessarily) of nonelliptic elements. Then, by (3.5), $\tilde{\Gamma} \cap \phi^{-1}\left(\Psi_{I}\right), i=1,2$, each contains hyperbolic elements of exponential type. This is impossible by (6.2).

So one of $p_{1}, p_{2}$, say $p_{1}$, is an isomorphism. Setting $\Phi=p_{1}(\Gamma)$ and $\rho=p_{2} \circ p_{1}^{-1}$ we have $\Gamma=\Phi_{\rho}$. The last assertion of part (3)(ii) is clear by previous arguments.

(7.1.1) Remark. By (7.1) and (5.2), and trivially if $\Gamma \approx \mathbf{Z} \approx \mathbf{Z}$, or $\mathbf{Z}^{2}$, we see that we always have $\Gamma=\Phi_{\rho}$ or $\left\langle\Phi_{\rho}, \Psi_{2}\right\rangle$. All these cases with $\tilde{\Gamma}$ discrete but not necessarily properly discontinuous do occur. An interesting example of this type is $\Phi$ with $\Phi=a$ discrete subgroup of $P_{1}$ such that $\Phi \approx \pi_{1}$ (a closed surface of genus $\geqslant 2$ ) and $\rho: \Phi \rightarrow P_{1}$ faithful with discrete image. Then $\phi^{-1}\left(\Phi_{\rho}\right) \approx \Phi \times \mathbf{Z}$ ! In fact, there is no known example of $\Gamma=\Phi_{\rho}$ acting properly discontinuously on $S_{1}, \Phi$ a nonabelian free group, and image of $\rho$ not contained in a group conjugate to $K$.

(7.2) Theorem. Let $M=\tilde{\Gamma} \backslash S_{\infty}$ be a compact space-form. Then $M$ has finite level.

Proof. Suppose not. By passing to a finite covering we may assume that $\tilde{\Gamma}$ is contained in $I_{0}\left(S_{\infty}\right)$. Then $h=$ the generator of $Z$ centralizes $\tilde{\Gamma}$ and induces a Lorentz isometry $\bar{h}$ of $M$. Let $G$ be the full group of Lorentz isometries of $M$. It is well known (cf. [29]) that $G$ is a Lie group. First assume that $\langle\bar{h}\rangle$ is not a discrete subgroup of $G$. Let $C$ be the identity component of its closure. Clearly $C$ has dimension $\geqslant 1$. Let $\tilde{C}$ be the identity component of the group induced by $C$ on $S_{\infty}$. Then $\tilde{C}$ is contained in $Z_{I_{0}\left(S_{\infty}\right)}(\tilde{\Gamma})$, and we are in the situation described at the beginning of the proof of (7.1). The possibilities (2) and (3) there only involve groups of cohomological dimension $\leqslant 2$. But since $M$ is compact the cohomological dimension of $\tilde{\Gamma}$ is 3 . This contradiction shows that $M$ is of finite level-and so $\langle\bar{h}\rangle$ is indeed finite.

Now we claim that $\langle\bar{h}\rangle$ cannot be an infinite discrete subgroup of $G$. Indeed $h$ is a Lorentz isometry and so preserves the associated volume form. Since $M$ is compact, its volume is finite. So by the well-known recurrence theorem of Poincaré, cf. for example [24], almost all points are recurrent, i.e., for $x$, not in a nowhere dense set of measure $0, \bar{h}^{n}(x)$ is close to $x$ for infinitely many integers $n$. Now if we coordinatize $S_{\infty}$ as a product $\tilde{D} \times \tilde{K}$ where $\tilde{D}$ is a lift of the disk of a conjugacy class of elliptics in $P_{1}$, then in these coordinates $h$ acts as a translation in the $\tilde{K}$-factor. So in these coordinates $h_{*} \equiv 1$. In other words, if $\bar{h}^{n}(x)=y$ is close to $x$ and $\tau$ is the parallel translation along a geodesic from $x$ to $y$, then $\bar{h}_{*}^{n}$ is close to $\tau_{*}$. So, since $M$ is compact, $\bar{h}^{n}$ is close to identity i.e. $\langle\bar{h}\rangle$ cannot be discrete unless it is finite. This finishes the proof. 
(7.3) Corollary. A compact Lorentz orbifold has a finite (orbifold) covering which is homeomorphic to a circle bundle over a closed surface of genus $\geqslant 2$.

Proof. Let $M$ be a compact Lorentz orbifold. It has a finite orbifold covering $M_{1}$ which is a compact Lorentz space-form with $\pi_{1}\left(M_{1}\right) \approx \tilde{\Gamma} \subseteq$ $I_{0}\left(S_{\infty}\right)$, so that by (7.2) and (5.2) the image $\Gamma$ of $\tilde{\Gamma}$ in $I_{0}\left(S_{1}\right)$ is $\Phi_{\rho}$ for a suitable discrete cocompact subgroup $\Phi$ of $P_{1}$. By passing to another finite covering if necessary we may assume that $\Phi$ is torsion free, hence $\approx \pi_{1}$ (closed surface of genus $\geqslant 2$ ). The result now follows by a well-known result of Waldhausen, cf. [36].

(7.4) Remark. Let $M=\tilde{\Gamma} \backslash S_{\infty}$ be a compact Lorentz space-form so $\tilde{\Gamma}$ is torsion free. Then, $M$ is homeomorphic to a closed 3-manifold. If we moreover assume that $M$ is Haken in the sense of 3-dimensional topology, cf. [6], then by [36] it follows that $M$ itself is a Seifert fiber space and in fact, the hypothesis that $M$ is Haken may also be dropped by the recent result of Scott, cf. [30]. This structure of a Seifert fiber space on $M$ may be chosen so that the Lorentz isometries which yield the covering $M_{1} \rightarrow M$ are, up to an equivalence of covering spaces, isomorphisms of a perhaps deformed Seifert structure on $M_{1}$. This last fact is not obvious and depends on some subtleties in the Seifert construction in the framework of [3]. Moreover, if we do not assume $\tilde{\Gamma}$ to be torsion-free so that $M=\tilde{\Gamma} \backslash S_{\infty}$ is only a Lorentz orbifold we may still characterize the topology of $M$. By a recent unpublished result of Meeks and Scott, cf. [17] such compact $M$ is necessarily a Seifert orbifold (to be defined more precisely in the next section). Unfortunately, all this uses recently developed deep 3-dimensional topology and still does not relate the Lorentz structure to the Seifert structure. In the standard Lorentz orbifolds, studied in the next section the relationship between the Lorentz structure and a "naturally preferred" Seifert structure is clear.

We note however that a structure of a Lorentz orbifold on a large class of closed Seifert fiber spaces must be standard as the following result shows.

(7.5) Proposition. Let $M=\tilde{\Gamma} \backslash S_{\infty}$ be a Lorentz orbifold such that $\tilde{\Gamma} \subseteq$ $\left\langle I_{0}\left(S_{\infty}\right), i_{u_{0}}\right\rangle$ and $\Gamma=\phi(\tilde{\Gamma})$ has a subgroup $\Gamma_{1} \subseteq I_{0}\left(S_{1}\right)$ of finite index with nontrivial finite center. Then $M$ is standard. In particular, if $M$ is covered by $M_{1}=$ a circle bundle over a closed orientable surface of genus $g \geqslant 2$ whose Euler number $b$ does not divide $2 g-2$, then $M$ is standard.

Proof. Indeed if $z \in Z\left(\Gamma_{1}\right), z \neq e$, then up to a suitable conjugation, $z \in K \times K$ and $\Gamma_{1} \subseteq P_{1} \times K$. So $\tilde{\Gamma}$ is standard by (2.8). As for the latter statement note that $\pi\left(M_{1}\right)$ has a presentation:

$$
\left.\pi_{1}\left(M_{1}\right)=\left\langle a_{i}, b_{i}, t\right| \prod_{i=1}^{g}\left(a_{i}, b_{i}\right)=t^{b}, t \text { central }\right\rangle .
$$


Identifying $\pi_{1}\left(M_{1}\right)$ with a subgroup of $I_{0}\left(S_{\infty}\right)$ we see that the image $\bar{t}$ of $t$ in $I_{0}\left(S_{1}\right)$ is of finite order by (7.2), and $\bar{t} \neq e$ since $b+2 g-2$. So $M_{1}$ and hence $M$ are standard by (2.8).

In view of $\S 8,[28]$ and $\S 10$, we note that if $M$ is a nonhomogeneous compact space-form with $e\left(M_{1}\right) \neq 0$, then $M$ must be standard.

(7.6) Remark. It is not difficult to see that (7.2) cannot be extended to space-forms of finite volume.

\section{Standard Lorentz orbifolds and Seifert orbifolds}

(8.1). In this section we shall restrict to subgroups $J_{0}$ and $J$ of $I_{0}\left(S_{\infty}\right)$, cf. (2.4) and (2.5). By a standard Lorentz orbifold we mean one whose group is conjugate to a subgroup of $J$. Since $J$ acts properly on $S_{\infty}$, the standard Lorentz orbifolds just correspond to discrete subgroups of $J$. We shall relate the standard orbifolds to Seifert orbifolds which are constructed purely topologically and which, for the purposes of this paper, are defined as follows. Let $M$ be a Seifert fiber space in the usual sense cf. [32], [20], or [7], cf. also [14] for an elementary intuitive account. For simplicity, we restrict to the case when $\pi_{1}(M)$ is finitely generated. Let $G$ be a finite group of fiber-preserving homemorphisms of $M$, and $\tilde{G}=$ the group of all lifts of elements of $G$ to the universal cover $\tilde{M}$. A space of the type $H \backslash \tilde{M}$, or more precisely the triple ( $\tilde{M}, H, H \backslash \tilde{M})$, where $H$ is a subgroup of $\tilde{G}$ will be called a Seifert orbifold. Thus the fibers are homeomorphic to $S^{1}$, or $\mathbf{R}$, or a closed or halfclosed interval. The orbit-space (obtained by identifying each fiber to a point) is a 2-manifold possibly with boundary. Moreover, exactly as in the usual case of Seifert fiber spaces, if $H$ does not contain $\mathbf{Z}$ or $\mathbf{Z}^{2}$ as a subgroup of finite index then the orbit-space admits a structure of a 2-dimensional spherical, Euclidean or hyperbolic orbifold. This structure on the orbit-space is not necessarily unique, but the type of the geometry is determined by $H$ itself. The general theory of Seifert orbifolds is contained in that of "Seifert construction" in [15], which, to our knowledge appears for the first time in [3]. It would lead us too far to explain this machinery and therefrom deduce our present case. We shall need its full force in [13] when we consider Lorentz orbifolds and their deformations of structure. Here, instead, we shall work more directly. This is possible, and indeed, is of special interest, because of the more precise knowledge in low dimensions. 
(8.2). We now explain how the difference between a Seifert fiberspace, and a Seifert orbifold shows up in their associated groups. The fundamental group of a closed Seifert fiber space of type (Oo) has a presentation, cf. [20],

$$
\left.\pi=\left\langle a_{i}, b_{i}, x_{j}, t\right| \prod_{i=1}^{g}\left(a_{i}, b_{i}\right) \prod_{j=1}^{u} x_{j}=t^{b}, x_{j}^{\alpha_{j}}=t^{-\beta_{j}}, t \text { central }\right\rangle,
$$

where

$$
\text { g.c.d. }\left(\alpha_{j}, \beta_{j}\right)=1 \text { for } j=1,2, \cdots, u \text {. }
$$

The subgroup $\langle t\rangle$ is central and

$$
(* * *) \quad \pi /\langle t\rangle \approx \Phi=\left\langle\bar{a}_{i}, \bar{b}_{i}, \bar{x}_{j} \mid \prod_{i=1}^{g}\left(\bar{a}_{i}, \bar{b}_{i}\right) \prod_{j=1}^{u} \bar{x}_{j}=\bar{x}_{j}^{\alpha_{j}}=e\right\rangle,
$$

which is a well-known presentation of a planar discontinuous group. The rational number

$$
\chi(\phi)=2-2 g-\sum_{j=1}^{u}\left(1-\frac{1}{\alpha_{j}}\right)
$$

is called the Euler characteristic of $\Phi$. As is well known $\chi(\Phi)>0,=0,<0$ according to whether $\Phi$ can be realized as a discrete group of isometries in the 2-dimensional spherical, Euclidean or hyperbolic geometry respectively. If $\chi(\Phi) \leqslant 0$ then $\langle t\rangle \approx \mathbf{Z}$ and (*) exhibits $\pi$ as a central extension of $\mathbf{Z}$ by $\Phi$ whose congruence class defines an element of $H^{2}(\Phi ; \mathbf{Z})$ cf. [16, Chapter 4]. Notice that if $\chi(\Phi)<0$ then $Z(\pi)=\langle t\rangle$ and $\Phi$ is canonically associated with $\pi$. Now every central Z-extension of $\Phi$ clearly has a presentation of the type (*), but $(* *)$ precisely picks up the torsion-free ones. The groups $\pi$ defined by $(*)$ with $\chi(\Phi)<0$ but not necessarily satisfying $(* *)$ are precisely the "fundamental groups of closed Seifert orbifolds of type (Oo) with hyperbolic base".

We should remark that when the fibers are all circles, the underlying topological space of a Seifert orbifold is also a Seifert fiber space. But the "branching", which is reflected in the torsion in the group, is an extra invariant of the structure.

Similarly

$$
(*)^{\prime} \quad \pi=\left\langle a_{i}, x_{j}, t \mid \prod_{i=1}^{k} a_{i}^{2} \prod_{j=1}^{u} x_{j}=t^{b}, x_{j}^{\alpha_{j}}=t^{-\beta_{j}}, x_{j} t=t x_{j}, a_{i} t=t^{-1} a_{i}\right\rangle
$$


are the groups of closed Seifert orbifolds of type (On)

$$
\begin{gathered}
(*)_{0} \quad \pi=\left\langle a_{i}, b_{i}, x_{j}, y_{k}, t\right| \prod_{i=1}^{g}\left(a_{i}, b_{i}\right) \prod_{j=1}^{u} x_{j} \prod_{k=1}^{v} y_{k}=t^{b}, x_{j}^{\alpha_{j}}=t^{-\beta_{j}} \\
t \text { central }\rangle \\
(*)_{0}^{\prime} \quad \pi=\left\langle a_{i}, x_{j}, y_{k}, t\right| \prod_{i=1}^{k} a_{i}^{2} \prod_{j=1}^{u} x_{j} \prod_{k=1}^{v} y_{k}=t^{b}, x_{j} t=t x_{j}, \\
\left.x_{j}^{\alpha_{j}}=t^{-\beta_{j}}, y_{k} t=t y_{k}, a_{i} t=t^{-1} a_{i}\right\rangle
\end{gathered}
$$

with $v>0$ are the groups of nonclosed Seifert orbifolds of type $(\mathrm{Oo})$ and $(\mathrm{On})$ resp., with all fibers $\approx S^{1}$ where throughout we are restricting to finitely generated ones. If a Seifert orbifold is obtained from a Seifert fiber space by factoring out by a finite group containing a reflection, i.e., an orientation preserving involution which is orientation reversing on the base and the fiber, and having a fixed point on the base, then the base of the orbifold is a 2-manifold with boundary, and the fibers over the boundary are closed intervals. The groups of such orbifolds, if infinite, are again extensions of $\mathbf{Z}$ by crystallographic groups in the plane Euclidean or hyperbolic geometry. The presentations of these groups are complicated cf. [39, p. 119], but actually straightforward once one notes that the topology of the base, and the branching data, uniquely determine these Seifert orbifolds.

Since $J$ is orientation-preserving on $S_{\infty}$, the standard Lorentz orbifolds are orientable, so we shall not discuss nonorientable Seifert orbifolds and their groups.

(8.3). Now we describe the structure of discrete subgroups of $J_{0}$ and $J$. We use the notations of $\S 4$. Notice that $\phi\left(J_{0}\right)=P_{1} \times K$ and $\phi(J)=\left(P_{1} \times K\right)$ $\rtimes\left\langle i_{u_{0}}\right\rangle$.

Proposition. Let $\tilde{\Gamma}$ be a finitely generated discrete subgroup of $J_{0}$ such that $\tilde{\Gamma}$, $\Gamma, \Gamma_{1}, \Gamma_{2}$ are all torsion free. Then either

(1) $\Gamma=\Phi_{\rho}$ where $\Phi$ is a discrete subgroup of $P_{1}$ and $\rho: \Phi \rightarrow K$ a representation or

(2) $\Gamma$ is abelian but not discrete with the following possibilities:

(i) $\Gamma=\langle(x, y)\rangle x, y$ both elliptic and one of them is of infinite order;

(ii) $\Gamma \approx \mathbf{Z}^{2}$ contained in $B \times e$ where $B$ is conjugate to $A$ or $N$, or else contained in $B \times K$ where $B$ is conjugate to $A$ or $N$; or

(3) $\Gamma=\left\langle\Phi_{\rho}, \Psi_{2}\right\rangle \approx \Phi \times \Psi_{2}$ where $\Phi$ is a discrete nonabelian free subgroup of $P_{1}, \rho: \Phi \rightarrow K$ a representation, and $\Psi_{2}=\langle y\rangle$ with y elliptic of infinite order. 
Moreover, if $\tilde{\Gamma}$ has finite level then (1) occurs, and if $\tilde{\Gamma}$ is cocompact then (1) occurs with $\Phi \approx \pi_{1}$ (a closed surface of genus $\geqslant 2$ ).

Proof. Use (4.2), (5.2), and (7.2). Actually if $\tilde{\Gamma}$ is cocompact (and contained in $J_{0}$ ) then (7.1) also suffices to ensure that it has finite level.

(8.3.1) Remark. Let $\tilde{\Gamma}$ be an arbitrary finitely generated subgroup of $J$. Then $\tilde{\Gamma}$ contains a normal subgroup $\Gamma^{\prime}$ of finite index which has the structure described in (8.3). From this, actually, the structure of $\tilde{\Gamma}$ itself can be described completely. For instance if $\tilde{\Gamma}$ has level 1 then $\Gamma_{0}=\Gamma \cap \phi\left(J_{0}\right)$ has the form $\left\langle\Phi_{\rho}, \Psi_{2}\right\rangle$ where $\Phi$ is a discrete subgroup of $P_{1}, \rho: \Phi \rightarrow K$ a representation, and $\Psi_{2}$ a finite subgroup of $e \times K$. If $\Gamma_{0} \neq \Gamma$ then $\Gamma=\left\langle\Gamma_{0}, \gamma\right\rangle$ where $\gamma \in P_{1} \rtimes\left\langle i_{u_{0}}\right\rangle$ which normalizes $\Gamma_{0}$. A detailed description of $\tilde{\Gamma}$ 's with higher level can be based on [28, Theorem 1]. Finally, notice that in all cases of $\tilde{\Gamma}, p_{1} \circ \phi(\tilde{\Gamma})$ is discrete, except when $\tilde{\Gamma} \approx \mathbf{Z}$ as in (2)(i) with $x$ of infinite order or $\tilde{\Gamma}^{\prime} \approx \mathbf{Z}^{2}$ with $\tilde{\Gamma}^{\prime}$ contained in $B \times e$ with $B$ conjugate to $A$ or $N$. In any case $\tilde{\Gamma}$ is isomorphic to a group of a Seifert orbifold with hyperbolic base. This may be more apparent to the reader if one recalls that $\tilde{\Gamma} \cap \mathbf{R}$ will be discrete and that $\tilde{\Gamma} / \tilde{\Gamma} \cap \mathbf{R}=\boldsymbol{\Phi}=\left(p_{1} \circ \phi\right)(\tilde{\Gamma})$. The Seifert orbifold structure on $\tilde{\Gamma} \backslash S_{\infty}$ now descends from the normal $\mathbf{R}$ subgroup of $J$ and actually factors through $(\tilde{\Gamma} /(\tilde{\Gamma} \cap \mathbf{R})) \backslash S_{\infty}$.

(8.4). To describe precisely the relationship between standard Lorentz orbifolds and Seifert orbifolds we need the notion of the "Euler number of a Seifert orbifold". For Seifert fiber spaces this notion was defined in [23]. The notion is significant for closed Seifert orbifolds of type $(O o)$ and $(O n)$. For an orbifold $M$ of this type with the corresponding group as in $(*)$ or $(*)^{\prime}$ in (8.2) set

$$
e(M)=e(\pi)=-\left(b+\sum_{j=1}^{u} \beta_{j} / \alpha_{j}\right) .
$$

If $\pi$ contains reflections, let $\pi_{0}$ be the subgroup of $\pi$ of index 2 which centralizes $\langle t\rangle$. Then set $e(\pi)=\frac{1}{2} e\left(\pi_{0}\right)$. The significance of the notion is that it depends only on the congruence class in $H^{2}(\Phi ; \mathbf{Z})^{3}$ represented by $\pi$ and not on the specific presentation given in $(*)$ or $(*)^{\prime}$. If there is no "branching" i.e. no $x_{j}$ 's or $\left(\alpha_{j}, \beta_{j}\right)$ 's, the Seifert orbifold is a circle bundle over a closed surface, and $e(\pi)=-b$ which is just the Euler- or Chern-number of this circle bundle. If $\pi^{\prime}$ is a subgroup of finite index in $\pi, \pi^{\prime} \cap\langle t\rangle=\left\langle t^{m}\right\rangle$, and the image of $\pi^{\prime}$ in $\Phi$ has index $n$ in $\Phi$, then $e\left(\pi^{\prime}\right)=n e(\pi) / m$. The proof of this fact is similar to the one in [23] for the case of Seifert fiber spaces. If $M$ is nonclosed then set $e(M)=e(\pi)=0$.

\footnotetext{
${ }^{3}$ Note that $\mathbf{Z}$ is a nontrivial $\Phi$-module iff $\Phi$ contains reflections or glide reflections.
} 
(8.5) Theorem. (a) (Structure). Let $M=\tilde{\Gamma} \backslash S_{\infty}$ be a standard Lorentz orbifold then $M$ is an orientable Seifert orbifold with hyperbolic base. Moreover, if $M$ is closed then $e(M) \neq 0$. Conversely,

(b) (Realization). Let $M$ be an orientable Seifert orbifold with all fibers $\approx S^{1,4}$ and hyperbolic base. ${ }^{4}$ Moreover, if $M$ is closed assume that its Euler number is $\neq 0$. Then $M$ admits a structure of a standard Lorentz orbifold.

Proof. (a) First notice that by (8.3.1), $\Gamma$ is a Seifert orbifold group with hyperbolic base $\Phi=p_{1} \circ \phi(\Gamma)$, so $e(\Gamma)$ makes sense. The orbits of $e \times \tilde{K}$ in $J_{0}$ on $S_{\infty}$ project down on $M$ and give it a structure of a Seifert orbifold. Now suppose that $M$ is closed. Using the multiplicative properties of $e(M)$ noted in (8.4), and passing to a finite cover if necessary, we may assume that $\tilde{\Gamma}$ satisfies the hypotheses of (8.3). So $\Phi \approx \pi_{1}$ (closed surface of genus $g \geqslant 2$ ). Then the calculation similar to the one at the end of (4.2) shows that $e(\tilde{\Gamma})$ is a nonzero rational multiple of $2 g-2 \neq 0$. This proves (a).

(b) First consider the case when $M$ is closed with $\pi$ as in (*), cf. (8.2). We first realize $\pi$ as a discrete subgroup of $J_{0}$. Note that $\chi(\Phi)<0$, and by assumption $e(\pi) \neq 0$. So $\rho=e(\pi) / \chi(\Phi) \neq 0$. First realize $\Phi$ as in (***), cf. (8.2) as a discrete subgroup in $P_{1}$. Then it is possible to choose the generators $\bar{a}_{i}, \bar{b}_{i}, \bar{x}_{j}$ of $\Phi$ and their lifts $a_{i}, b_{i}, x_{j}$ to $P_{\infty}$ so that $x_{j}^{\alpha_{j}}=t$ and $\Pi\left(a_{i}, b_{i}\right) \Pi x_{j}$ $=t^{2 g-2+u}$, cf. [28, Theorem 1]. Geometrically, $x$ acting on the hyperbolic plane has a fixed point, and is a rotation through $2 \pi / \alpha_{j}$ around the fixed point. Now let

and set (in the notation of §2)

$$
r_{j}=\frac{1}{\alpha_{j}}+\frac{1}{\rho} \frac{\beta_{j}}{\alpha_{j}}
$$

$$
\begin{aligned}
& A_{i}=\left[\left(a_{i}, e\right)\right], \quad B_{i}=\left[\left(b_{i}, e\right)\right], \\
& X_{j}=\left[\left(x_{j}, t^{r_{j}}\right)\right], \quad T=\left[\left(e, t^{-1 / \rho}\right)\right] \text {. }
\end{aligned}
$$

Then

and similarly

$$
\begin{aligned}
X_{j}^{\alpha_{j}} & =\left[\left(x_{j}^{\alpha_{j}}, t^{r_{j} \alpha_{j}}\right)\right]=\left[\left(t, t^{1+1 / \rho \beta_{j}}\right)\right] \\
& =\left[\left(e, t^{1 / \rho \beta_{j}}\right)\right]=T^{-\beta_{j}},
\end{aligned}
$$

$$
\prod_{i=1}^{g}\left(A_{i}, B_{i}\right) \prod_{j=1}^{u} X_{j}=T^{b} .
$$

One easily checks, starting from the fact that $\Phi$ is a discrete subgroup of $P_{1}$, that $\left\{A_{i}, B_{i}, X_{j}, T\right\}$ define a discrete faithful representation of $\pi$ in $J_{0}$.

\footnotetext{
${ }^{4}$ These hypotheses are made for simplicity only. In the next section we deal with the general case.
} 
Realizing $\pi$ in $J$ in the case of $(*)^{\prime}$ cf. (8.2) is similar. Here we have

$$
\Phi=\left\langle\bar{a}_{i}, \bar{x}_{j} \mid \prod_{i=1}^{k} \bar{a}_{i}^{2} \prod_{j=1}^{u} \bar{x}_{j}=e=\bar{x}_{j}^{\boldsymbol{\alpha}_{j}}\right\rangle
$$

and $\chi(\Phi)=2-k+\sum_{j=1}^{u}\left(1-1 / \alpha_{j}\right)$. Set $\rho=e(\pi) / \chi(\Phi)$, realize $\Phi$ as a discrete subgroup in $P_{1} \rtimes\left\langle i_{u_{0}}\right\rangle$, and proceed exactly as before.

Realizing $\pi$ in the class $(*)_{0}$ and $(*)_{0}^{\prime}$ in $J$ is analogous, and simpler-take $\rho$ to be any nonzero number, e.g. 1 , and proceed as before.

Let $\pi \backslash S_{\infty}=N$. We still have to ensure that $N \approx M$ as Seifert orbifolds. To start with, $N$ does have a structure of a Seifert orbifold where the fibers are the projections of the orbits of $e \times \tilde{K}$ in $J_{0}$ on $S_{\infty}$. First we must check the Seifert invariants of the singular fibers. Let $C$ be a singular fiber, and $\tilde{C}$ its lift in $S_{\infty}$. For computational purposes identify $S_{\infty}$ with $P_{\infty}$ so that $e$ is on $\tilde{C}$, and its projection in the hyperbolic plane is fixed by $x_{j}$. Hence $\tilde{C}=e \times \tilde{K}$, and it is left invariant by $X_{j}$. Parametrize $\tilde{K}$ by $\mathbf{R} \ni y \rightarrow t^{y / \rho}$. By construction we have $x_{j}=t^{1 / \alpha_{j}}$, and so

$$
X_{j}\left(t^{y / \rho}\right)=t^{1 / \alpha_{j}+y / \rho-r_{j}}=t^{\left(y-\beta_{j} / \alpha_{j}\right) / \rho} .
$$

In other words, the action of $X_{j}$ on $\tilde{C}$ is, in terms of the parameter $y, y \rightarrow y-$ $\beta_{j} / \alpha_{j}$. This precisely means that the singular fiber $C$ has Seifert invariants $\left(\alpha_{j}, \beta_{j}\right)$. Topologically, what has happened in passing from the inverse image $\pi_{0}$ of $\Phi$ in $P_{\infty}$ to $\pi$ in $J$ is that, $\pi \backslash S_{\infty}$ is obtained from $\pi_{0} \backslash S_{\infty}$ by replacing a fibered solid torus with invariants $\left(\alpha_{j},-1\right)$ by a fibered solid torus with invariants $\left(\alpha_{j}, \beta_{j}\right)$. When $N$ is nonclosed we have to be careful in choosing $\Phi$ in the first place, so that the genus of the orbitspace of $\pi_{0} \backslash S_{\infty}$ is the same as that of the orbit-space of $M$. With this data in hand one can construct a fiber-preserving homeomorphism of $N$ onto $M$ as in the original argument of Seifert, cf. [32] in the case of Seifert fiber spaces.

(8.6). Finally we note the following interesting relationship between the topology and geometry of a closed standard Lorentz orbifold.

Theorem. The Lorentz volume of a closed standard Lorentz orbifold is an invariant of the underlying Seifert orbifold structure. This volume is a rational multiple of $4 \pi^{2}$.

Proof. Given a closed standard orbifold $M$ with $\pi$ as in $(*)$ or $\left(*^{\prime}\right)$, the rational number $v(M)=(\chi(\Phi))^{2} /|e(\pi)|$ is certainly an invariant of the Seifert orbifold structure. Let $\pi^{\prime}$ be a subgroup of finite index in $\pi$ and let $\Phi^{\prime}$ be its image in $\Phi$. If the index of $\Phi^{\prime}$ in $\Phi$ is $n$ and $\pi^{\prime} \cap\langle t\rangle=\left\langle t^{m}\right\rangle$ it is clear that the index of $\pi^{\prime}$ in $\pi$ is $m n$. Let $M^{\prime}$ be the branched cover of $M$ corresponding to $\pi^{\prime}$. From the multiplicative properties $\chi\left(\Phi^{\prime}\right)=n \chi(\Phi)$ and 
$e\left(\pi^{\prime}\right)=n e(\pi) / m$ it follows that $v\left(M^{\prime}\right)=m n v(M)$. Now if $M$ is the unit tangent circle bundle of a surface of genus $g \geqslant 2$ its Lorentz volume is easily computed to be $4 \pi^{2}(2 g-2)=4 \pi^{2}|\chi(\Phi)|=4 \pi^{2} \chi(\Phi)^{2} /|e(\pi)|$ since in this case $e(\pi)=-\chi(\Phi)$. Since in passing to a branched cover the volume gets multiplied by the degree, it follows that the Lorentz volume of a closed standard Lorentz orbifold is $4 \pi^{2} v(M)$. This finishes the proof.

(8.6.1) Remark. By contrast, one should note that the volume of a nonclosed standard Lorentz orbifold, even among those with finite volumes, is not an invariant of the orbifold structure.

\section{Topology of standard Lorentz orbifolds}

(9.1). The quotients of 3-manifolds by orientation-preserving properly discontinuous groups are again manifolds. So the standard Lorentz orbifolds are topologically again manifolds. We shall now identify their topological types thus complementing (8.5), (8.6), and completing the proof of the theorem on standard orbifolds stated in the introduction. For brevity, we formulate the theorem in the closed case, but during the course of the proof we shall make some comments on the nonclosed cases. Recall that a reflection in $J$ is an involution reversing space- and time-orientations, whereas a reflection on a 2-manifold is an orientation-reversing involution which has a 1-dimensional fixed point set.

Theorem. Let $M=\tilde{\Gamma} \backslash S_{\infty}$ be a closed standard orbifold. (i) If $\tilde{\Gamma}$ does not contain reflections then $M$ is a closed orientable Seifert fiber space with nonzero Euler number. Conversely a closed orientable Seifert fiber space with nonzero Euler number admits the structure of a standard Lorentz orbifold of the type $\tilde{\Gamma} \backslash S_{\infty}$ with $\tilde{\Gamma}$ containing no relfections. (ii) If $\tilde{\Gamma}$ contains reflections then $M$ is homeomorphic to a connected sum of lens spaces (including $S^{2} \times S^{1}$ and $S^{3}$ ). Conversely every such connected sum admits the structure of a Lorentz orbifold of the type $\tilde{\Gamma} \backslash S_{\infty}$ with $\tilde{\Gamma}$ containing reflections.

Proof. (i) Suppose that $\tilde{\Gamma}$ contains no reflections. We have already seen that $\tilde{\Gamma} \backslash S_{\infty}=M$ is a Seifert orbifold whose Seifert structure descends from the $\mathbf{R}$-action on $S_{\infty}, \mathbf{R} \subset J_{0}$, to yield closed time-like geodesics on $M$. The fibers are all circles if $\tilde{\Gamma}$ contains no reflections. As such, the map which collapses each fiber to a point $M \rightarrow \Phi \backslash H, \Phi=\tilde{\Gamma} /(\tilde{\Gamma} \cap \mathbf{R})$ is a Seifert fibering over a hyperbolic base in its own right. If we let $N$ denote $M$ as a Seifert fiber space we may determine easily the Seifert invariants of $N$ from $\tilde{\Gamma}$. If a "singular" fiber of the orbifold $M$ has Seifert invariants $\left(\alpha_{j}, \beta_{j}\right)$ then this fiber, interpreted in $N$, will have Seifert invariant $\left(\alpha_{j}^{\prime}, \beta_{j}^{\prime}\right)$ where we have divided $\alpha_{j}$, and 
$\beta_{j}$ by the g.c.d. $\left(\alpha_{j}, \beta_{j}\right)$. In this way it is possible for a "singular" fiber of $M$ to become an ordinary fiber of $N$. The " $b$ " invariant associated with $M$, if it is relevant, will ordinarily be different from that of $N$. However still $e(\tilde{\Gamma})=e(N)$. In particular $e(N) \neq 0$.

It remains to check, for this situation, that every Seifert fiber space $N$ also has the structure of a standard Lorentz orbifold provided that $N$ is of type $(O o)$ or $(O n)$ and $e(N) \neq 0$, if $N$ is closed. We will achieve this by direct construction.

Recall the presentation of the fundamental groups of the Seifert fiber spaces of types $(\mathrm{Oo})$ and $(\mathrm{On})$ as given in 8.2. Since all $N$ 's can be realized as Lorentz space-forms by the construction in 8.5 if $v \neq 0$, we need only concern ourselves about the closed case. We shall begin with a presentation of $\pi_{1}(N)$ and add carefully generators and relators and alter the long relation of $\pi_{1}(N)$ to produce a new group $\tilde{\Gamma} \subset J$, even when $\pi_{1}(N)$ itself may not be realizable in $J$, provided that $e(N) \neq 0$. We shall then observe that $M=\tilde{\Gamma} \backslash S_{\infty}$ is homeomorphic to $N$ and the resulting Seifert invariants on $M$ are equivalent to those for $N$. Thus, the Seifert structure on $M$ descending from the R-action on $S_{\infty}$, via the branched covering map $S_{\infty} \rightarrow \tilde{\Gamma} \backslash S_{\infty}$, is equivalent to the prescribed Seifert structure on $N$.

There are many ways to construct different $\tilde{\Gamma}$ 's. Here is one method. We begin with a presentation for $\pi=\pi_{1}(N)$ using $(*)$ or $(*)^{\prime}$. To ensure that $\tilde{\Gamma}$ will embed in $J$ we add 3 generators to $\pi$ 's presentation, $z_{1}, z_{2}, z_{3}$ and the following relations $z_{1}^{\gamma_{1}}=t^{-\gamma_{1}}, z_{2}^{\gamma_{2}}=t^{-\gamma_{2}}, z_{3}^{\gamma_{3}}=t^{-\gamma_{3}}$, where $1 / \gamma_{1}+1 / \gamma_{2}+$ $1 / \gamma_{3}<1, \gamma_{i}>1$, and $t$ is to commute with the $z_{i}$ 's. We also want to alter the long relation to

$$
\prod_{i=1}^{g}\left(a_{i} b_{i}\right) \prod_{j=1}^{u} x_{j} z_{1} z_{2} z_{3}=t^{b-3} \text { or } \prod_{i=1}^{k} a_{i}^{2} \prod_{j=1}^{u} x_{j} z_{1} z_{2} z_{3}=t^{b-3} .
$$

Now the resulting group, $\tilde{\Gamma}$, consists of the generators of $\pi$ together with the $z_{i}$ and the added relations and altered long relation. Clearly, it is embeddable in $J$, provided $e(\tilde{\Gamma}) \neq 0$, since $(\tilde{\Gamma}) /\langle t\rangle=\Phi(\tilde{\Gamma})$ is now embeddable in $\operatorname{PSL}_{2}(2, \mathbf{R})$ $\rtimes \mathbf{Z}_{2}$ as a cocompact discrete group. We have constructed $\tilde{\Gamma}$ so that $e(\tilde{\Gamma})=$ $e(\pi)=e(N)$. In $\tilde{\Gamma}$ take the normal subgroup $\left\langle\left\langle z_{1}, z_{2}, z_{3}\right\rangle\right\rangle$ generated by $\left\{z_{1}, z_{2}, z_{3}\right\}$ and form the quotient $\tilde{\Gamma} /\left\langle\left\langle z_{1}, z_{2}, z_{3}\right\rangle\right\rangle$. We obtain the group $\pi$. In fact, dividing out by $\left\langle\left\langle z_{1}, z_{2}, z_{3}\right\rangle\right\rangle$ induces dividing out on $\Phi(\tilde{\Gamma})$ by the normal subgroup generated by the torsion elements $\left\{\bar{z}_{1}, \bar{z}_{2}, \bar{z}_{3}\right\}$ to yield $\Phi(\pi)$. (one may even check, when relevant, that the homomorphism $\Phi(\tilde{\Gamma}) \rightarrow \Phi(\pi)$ induced from $\tilde{\Gamma} \rightarrow \pi$ induces the cohomology homomorphism $H^{2}(\Phi(\pi) ; \mathbf{Z}) \rightarrow$ $H^{2}(\Phi(\tilde{\Gamma}) ; \mathbf{Z})$ which carries the extension class for $\pi$ onto that for $\tilde{\Gamma}$.) Conse- 
quently, $\tilde{\Gamma} \backslash S_{\infty}$ which will have a Seifert orbifold structure descending from $\mathbf{R} \subset J$ has Seifert invariants exactly equivalent to those of $N$. (Recall that the Seifert invariants corresponding to the $z_{i}$ are $\left(\gamma_{i}, \gamma_{i}\right)$ which is equivalent to $(1,1)$ and will be regarded as an ordinary fiber on $N$.) Since $e(\tilde{\Gamma})=e(\pi) \neq 0$, we have shown that $N$ also possesses an orbifold structure compatible with its Seifert structure.

(ii) Suppose that $\tilde{\Gamma}$ contains reflections. Then, $\Phi$ also contains reflections. Let $\Phi^{\prime} \subset \Phi$ be a normal subgroup of index 2, which preserves orientation on $H$ and $\tilde{\Gamma}^{\prime}=\phi^{-1}\left(\phi^{\prime}\right)$. Then $M=\tilde{\Gamma} \backslash S_{\infty}$ is just the quotient of $\tilde{\Gamma}^{\prime} \backslash S_{\infty}$ by the action of $\tilde{\Gamma} / \tilde{\Gamma}^{\prime} \cong \mathbf{Z}_{2}$ which is normalizing, and not centralizing, the induced $S^{1}$-action on the Seifert orbifold $M^{\prime}=\tilde{\Gamma}^{\prime} \backslash S_{\infty}$. Now the involution on $M^{\prime}$ must map $S^{1}$-orbits to $S^{1}$-orbits of the same type. Moreover, $\Phi / \Phi^{\prime}$, induces on the orbit space $\Phi^{\prime} \backslash H$, a reflection across a 1-manifold. This means that the orbits in $M^{\prime}$ above this 1-manifold are left invariant and the $\mathbf{Z}_{2}$-action is just $\boldsymbol{\theta} \rightarrow-\boldsymbol{\theta}$ on each of these orbits. Thus each such orbit in $M^{\prime}$ is collapsed to an arc in $M$.

Suppose this 1-manifold separates $\Phi^{\prime} \backslash H$ into 2-components. Then $\Phi \backslash H$ has a section $\mathscr{S}$ into $\Phi^{\prime} \backslash H$. Therefore, we may now construct $M$ as follows. Over $\mathscr{S}$ in $M^{\prime}$ we have an oriented $S^{1}$-space $M^{\prime}(\mathscr{S})$ with invariant "boundary" components. $M$ is obtained from $M^{\prime}(\mathscr{S})$ by identifying each $S^{1}$-orbit of the "boundary" of $M^{\prime}(\mathscr{S})$ to its orbit under the $\mathbf{Z}_{2}$-action. Now we still have an $S^{1}$-action on $M$ minus the collection of arcs. The $S^{1}$-action will extend if we collapse each arc to a point. We show, in the first picture below, the collection of fibers in $M$ over a short interval in $\mathscr{S}$ which emanates transversally from the $\mathbf{Z}_{2}$-image of a nonsingular $S^{1}$-orbit in the boundary of $\mathscr{S}$. The second picture (see Figure 2) show the result of collapsing the arc fiber over the initial point of this interval to a point.
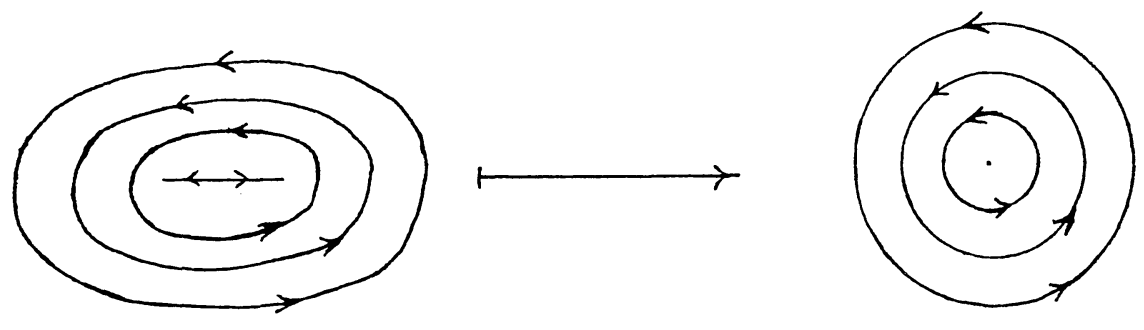

FIGURE 2

Obviously, the topological type of $M$ is unchanged. But now $M$ possesses a global $S^{1}$-action with fixed points. Such manifolds are built up as equivariant 
connected sums and are well understood, c.f. Theorems 2 and 4 of [27]. In the closed case $M$ is homeomorphic to an equivariant connected sum of lens spaces which include the possibility of $S^{2} \times S^{1}$ and the 3-sphere. In fact, we are exactly in the case (i) of Theorem 1 with $t=0$ of [21] or [20, p. 20]. In the open finitely generated case $M$ is the equivariant connected sum of the above together with the other basic open building blocks of $S^{1}$-actions with fixed points, $\mathbf{R}^{2} \times S^{1}$ and $\mathbf{R}^{2} \times \mathbf{R}^{1}$.

To handle the case where the $\mathbf{Z}_{2}$-action is across a 1-manifold which fails to separate we just observe that our analysis really was entirely local. The 1-manifold, of course locally separates, and so a neighborhood has a section and the argument just given above applies. However, there is one feature that does not appear in the global separating case. Namely, the involution on the complement will be orientation reversing and so the orbit space $\Phi \backslash H$ will be nonorientable. Moreover, over each orientation reversing curve, (containing no singular fibers), in the base we shall find in $M$ a one-sided Klein bottle. In the closed case the equivariant connected sum is still a connected sum of lens spaces including $S^{2} \times S^{1}$ and $S^{3}$ (Theorem 1, case (iv), with $t=0$ of [21]). Also, the open case is unchanged from the previous discussion.

It remains to check that all possibilities can be realized. As in the case of $\tilde{\Gamma}$ without reflections the realization can be done in many different ways. A reader familiar with the proof of Theorem 2 of [26] can easily construct such realizations.

First, we need the following fact. If $M^{\prime}$ is a Seifert 3-manifold of type (Oo) then one can always find orientation preserving involutions on $M^{\prime}$ which extends the $S^{1}$-action to an $O(2)$-action. To realize such an action, select an orientation reversing involution of the base which reflects, at least locally, across a 1-manifold. We arrange, by an isotopy, for all the singular orbits to lie above this 1-manifold in a specified order. Choose a section for the $S^{1}$-action away from invariant tubular neighborhoods of the singular orbits and, using the involution on the base, define the obvious orientation preserving involution which reflects the orientation of each $S^{1}$-orbit. Now the equivariant gluing map that attaches each fibered solid torus to the boundary tori commutes with this $\mathbf{Z}_{2}$ action which is generated by $\left(\begin{array}{rr}-1 & 0 \\ 0 & -1\end{array}\right)$ on the boundary tori. The $\mathbf{Z}_{2}$ action now extends over the solid fibered tori and together yields an $O(2)$ action on $M^{\prime}$. All of the $\mathbf{Z}_{2}$ orbits are free except for the two fixed points of each of the circles above the reflecting (or local reflecting) 1-manifold in the base. Thus, on $M=M^{\prime} / \mathbf{Z}_{2}$ we obtain an $S^{1}$-action or local $S^{1}$-action away from the arcs over the reflecting 1-manifold. Again we may collapse the arcs to points as in the description above and extend the action or local action so that 
these points are fixed. The homeomorphism type of the orbifold $M$ is unchanged. It is homeomorphic to $S^{3}$ connected sum with $2 g+h-1$ copies of $S^{2} \times S^{1}$, resp. $k+h-1$ copies of $S^{2} \times S^{1}$, where $g$ resp. $k$ is the genus of the $\mathbf{Z}_{2}$ orbit space of the base if it is orientable, resp. nonorientable, and $h$ is the number of connected components of the reflecting 1-manifold.

To obtain any number of specific lens spaces in this connected sum we alter the procedure slightly and arrange that some of the singular orbits of the same types in pairs be reflected to one another by the involution cf. [21]. In this way the $S^{1}$ or local $S^{1}$-action on $M$ has any number of components of fixed points and any number of specified singular orbits. Thus all the possibilities mentioned above have been constructed and exhibited as Seifert orbifolds. We realize $M$ as a standard Lorentz orbifold by embedding $\tilde{\Gamma}^{\prime}=\pi_{1}\left(M^{\prime}\right)$ into $J_{0}$ as in 8.5. Choose generators suitably so that the corresponding Fuchsian group $\Phi^{\prime}$ is normalized by an appropriate reflection. There, $\tilde{\Gamma}^{\prime}$ will be normalized by a reflection in $J$ and $\tilde{\Gamma}=\left\langle\tilde{\Gamma}^{\prime}\right.$, this reflection $\rangle$ with $M=\tilde{\Gamma} \backslash S_{\infty}$.

(9.2) Remarks. (i) The collapsing of the arcs to points yielded a new manifold $\bar{M}$ homeomorphic to $M$. On $\bar{M}$ we could extend a local $S^{1}$-action so that we were able to use the classification of $S^{1}$-manifolds to identify $M$ topologically. On the other hand, we should not lose sight of the Lorentz structure on $M$. The R-orbits on $S_{\infty}$ descend to time-like geodesics on $M$. These are all closed and simple except for the arcs. The travel along the exceptional descended lines is just back and forth across the arcs.

(ii) The closed Seifert 3-manifolds of types $(\mathrm{Oo})$ or $(\mathrm{On})$ which do not appear as standard Lorentz space-forms are:

(a) All $M$ 's so that $\pi_{1}(M)$ is finite;

(b) All other $M$ 's so that $\pi_{1}(M) /($ normal $\mathbf{Z})$ is Euclidean crystallographic. (That is, the quotient $\Phi$ contains $\mathbf{Z} \times \mathbf{Z}$ as a normal subgroup of finite index).

(c) All $M$ 's so that $e(M)=0$. (The condition $e(M)=0$ is equivalent to the existence of a global slice to the induced $S^{1}$-action, or equivalently, the element of $H^{2}(\Phi, \mathbf{Z})$ representing the extension of $\pi_{1}(M)$ is of finite order, or equivalently, rank $H_{1}(M, \mathbf{Z})$ is odd.)

The manifolds in (a) and (b) (with $e(M) \neq 0$ ) can appear as Lorentz orbifolds but those in (c) cannot, except for $S^{2} \times S^{1}$ and $\mathbf{R} P_{3} \# \mathbf{R} P_{3}$ for a rather perverse reason!

(iii) A complete description of all orbifolds for the remaining classical Seifert manifolds, including those with boundary, can be based on the methods of the section above.

(9.3). We close this section with some examples and illustrations.

(i) Let $M^{\prime}$ be an orientable Seifert manifold with base homeomorphic to $S^{2}$. After arranging the singular orbits to lie over the equator define, as in (9.2), an 
orientation and fiber preserving involution on $M^{\prime}$ which on the base $S^{2}$ reflects across its equator. The quotient $M=M^{\prime} / \mathbf{Z}_{2}$, as we have seen, must be $S^{3}$. The map $M^{\prime} \rightarrow M$ is a doubly branched covering and the branching is over a knot or link in $S^{3}$. This knot or link is precisely the image of the set of fixed points of our involution on $M^{\prime}$. Each orbit in $M^{\prime}$ over the equator contributes exactly 2 branch points. These knots and links are known as rational tangles and have been studied by numerous people. In particular, each closed Seifert manifold which is a $\mathbf{Z}_{2}$-homology sphere gives rise in this manner to a knot in $S^{3}$.

Any finite group of automorphisms of the Seifert structure of an integral homology sphere, or more generally of a Lorentz space-form over $S^{2}$ with all singular orbit types distinct, must leave invariant the singular orbits. Hence, it can be seen that it must be isomorphic to a subgroup of $O(2)$ described above. Thus, a finite subgroup is at most dihedral with a cyclic normal part imbedded in the $S^{1}$ action and the quotient $\mathbf{Z}_{2}$ inducing a reflection across a separating circle in the base. Consequently, the orbifold structure is easily calculated.

(ii) We have described a couple of techniques among many available for obtaining certain Seifert fiber spaces as Lorentz orbifolds. The nilmanifolds which are principal circle bundles with $e(M) \neq 0$ are Lorentz orbifolds but not Lorentz space-forms (because of 9.2(ii)(b)). If we choose $\Phi=$ $\left\{a, b, x \mid a b a^{-1} b^{-1} x=1, x^{\alpha}=1\right\}, \alpha>1$, we get a cocompact discrete group of $P_{1}$ for which $\Phi \backslash H$ is a 2-torus with one branch point of index $\alpha$. The central extensions of $\mathbf{Z}$ by $\Phi$ can be presented as $\tilde{\Gamma}=\left\{a, b, x, t \mid a b a^{-1} b^{-1} x=t^{b}\right.$, $x^{\alpha} t^{\beta}=1, t$ central $\}$, and correspond to the elements of $H^{2}(\Phi ; \mathbf{Z}) \cong \mathbf{Z}$. As long as $(\alpha, \beta)=1, \tilde{\Gamma}$ is a Lorentz space-form. If $(\beta, \alpha) \neq 1$, then the Lorentz structure has singularities and we have an orbifold structure but not a space-form structure. For example, if we choose $b=0$, and $\beta=k \alpha, k \neq 0$, $\tilde{\Gamma} \subset J_{0}$ by the construction of (8.5) and $\tilde{\Gamma} \backslash S_{\infty}$ is homeomorphic to the nilmanifold with Euler number $=-k$. These orbifolds correspond to $\alpha \mathbf{Z} \subset \mathbf{Z}$ $=H^{2}(\Phi ; \mathbf{Z})$. Another procedure for producing nilmanifolds as Lorentz orbifolds is to take $\tilde{\Gamma} \backslash S_{\infty}=M^{\prime}$ where $\beta=k \alpha+1, b$ arbitrary, $k$ arbitrary, and divide out the $\mathbf{Z}_{\alpha}$ action contained in the $S^{1}$-action on $M^{\prime}$. This action is not free it is $\alpha$-branched over the singular orbit. The singular orbit in $M^{\prime}$ projects to an ordinary orbit in $M=\mathbf{Z}_{\alpha} \backslash M^{\prime}$ and yields a Lorentz orbifold homeomorphic to a nilmanifold with $e(M)=-(\alpha(k+b)+1)$.

(iii) In the open case (or, if one prefers, the almost identical compact case with boundary) a Seifert manifold over hyperbolic base also admits different and interesting geometric structures of a Riemannian manifold of nonpositive curvature. This arises from embedding the group $\tilde{\Gamma}$ in $\left(P_{1} \rtimes \mathbf{Z}_{2}\right) \times\left(\mathbf{R}^{1} \rtimes \mathbf{Z}_{2}\right)$ 
as a discrete subgroup. $\tilde{\Gamma}$ also embeds as a discrete subgroup of $J$ to yield a Lorentz space-form.

The $(p, q)$ torus knot space is the complement in $S^{3}$ of a $(p, q)$ torus knot. It admits a natural Siefert and hence Lorentz structure over a hyperbolic base. The base is a once punctured sphere with 2 branch points of branching index $p$ and $q$. Only the $(3,2)$ torus knot is a homeogeneous space $(\operatorname{PSL}(2, \mathbf{Z}) \backslash \operatorname{PSL}(2, \mathbf{R}))$, cf. [28]. The other groups need to be embedded in $J_{0}$. The only finite groups of Seifert automorphisms are again subgroups of $O(2)$ with the cyclic normal subgroups embedded in the $S^{1}$-action. The resulting orbifolds will be open solid tori, other $S^{1}$-manifolds over the open disk with 2 or fewer branch points, and $\mathbf{R}^{3}$.

\section{Homogeneous space-forms}

(10.1). A space-form $M=\Gamma \backslash S_{\infty}$ is said to be homogeneous if the full group $G$ of Lorentz isometries of $M$ is transitive on $M$. Then $\tilde{G}=$ the subgroup of $I\left(S_{\infty}\right)$ which consists of all lifts of elements of $G$ is clearly transitive on $S_{\infty}$ and $\tilde{G}$ normalizes $\tilde{\Gamma}$. Conversely, if $\tilde{G}$ is transitive on $S_{\infty}, M$ is homogeneous. If $M$ is homogeneous, clearly $\tilde{G}_{0}$ is still transitive on $S_{\infty}$ and centralizes $\tilde{\Gamma}$. J. Wolf, cf. [37], [38] listed certain families of homogeneous pseudo-Riemannian space-forms of constant curvature $\neq 0$. It was pointed out in [10] that this list is not complete, in fact, the family described below does not appear there. However [10] described fundamental groups of homogeneous forms only in a general way. In the present case of 3-dimensional Lorentz space-forms one can actually classify the homogeneous ones. These include some interesting families which have appeared in quite different contexts. The classification actually is quite easy in the present setting. We return back to the notations of $\S 4$. Notice that there are canonical embeddings $x \rightarrow{ }^{\alpha}[(x, e)]$ and $x \rightarrow{ }^{\beta}[(e, x)]$ of $P_{\infty}$ into $I\left(S_{\infty}\right)$. These are conjugate in $I\left(S_{\infty}\right)$-although not so in $I_{0}\left(S_{\infty}\right)$.

Theorem. Let $M=\tilde{\Gamma} \backslash S_{\infty}$ be an orbifold. Then $M$ is homogeneous iff $\tilde{\Gamma}$ is conjugate to a subgroup of $\alpha\left(P_{\infty}\right)$. Moreover, $M$ is necessarily a space-form.

Proof. If $\tilde{\Gamma}$, modulo conjugacy, is contained in $\alpha\left(P_{\infty}\right)$, clearly $Z_{I\left(S_{\infty}\right)}(\tilde{\Gamma}) \supseteq$ $\beta\left(P_{\infty}\right)$ which is transitive on $S_{\infty}$. Hence $M$ is homogeneous. Conversely suppose $M$ is homogeneous, and let $C=Z_{I\left(S_{\infty}\right)}(\tilde{\Gamma})$. If $C_{0}$ is $I_{0}\left(S_{\infty}\right)$ then $\tilde{\Gamma} \subseteq Z \subseteq \alpha\left(P_{\infty}\right)$. So assume $C_{0} \neq I_{0}\left(S_{\infty}\right)$. Now assume that $\tilde{\Gamma}$ is contained in $I_{0}\left(S_{\infty}\right)$. If $\tilde{\Gamma}$, modulo conjugacy, is in $\alpha\left(P_{\infty}\right)$ we are done. If otherwise, there would exist $(x, y) \in \tilde{\Gamma}$ with $x$ and $y$ not central elements of $P_{\infty}$. Since $C_{0} \subset$ 
$Z_{I\left(S_{\infty}\right)}(x, y)$ one sees that $C_{0}$ cannot be transitive on $S_{\infty}$ because of dimensional considerations. Therefore $\tilde{\Gamma}$ must be, modulo conjugacy, in $\alpha\left(P_{\infty}\right)$. Since $P_{\infty}$ is torsion free so is $\tilde{\Gamma}$. Hence $M$ is necessarily a space-form.

In general, $\tilde{\Gamma}$ contains a normal subgroup, $\tilde{\Gamma}^{\prime}=\tilde{\Gamma} \cap I_{0}\left(S_{\infty}\right)$, of index at most 4. $C_{0}(\tilde{\Gamma}) \subset C_{0}\left(\tilde{\Gamma}^{\prime}\right)$ and so $\tilde{\Gamma}^{\prime} \subset \alpha\left(P_{\infty}\right)$. On the other hand, a straight forward computational check shows that $\tilde{\Gamma}=\tilde{\Gamma}^{\prime}$.

(10.1.1) Remark. Thus the homogeneous space-forms are in 1-1 correspondence with the conjugacy classes of discrete subgroups of $P_{\infty}$. The algebraic structure of the cocompact discrete subgroups of $P_{\infty}$ is described in [28, Theorem 1], and this description contains classification of the topological types of closed homogeneous space-forms.

\section{A strange space-form}

(11.1). In this section we shall describe one curious phenomenon which would never occur in any "Riemannian" space-form problem. Usually, often without explicit mention, one restricts to Hausdorff spaces, and indeed the "space-forms" a là Helmholtz-Klein-Killing-Clifford... associated to standard spaces with Riemannian geometry are Hausdorff. But in as much as a "space-form" means "a space with the same local geometry as some standard model space such that the development map of its universal cover onto the model space is a covering map", cf. [9], or [3, Chapter 3] this only ensures that the space-form is locally Hausdorff and need not be so globally. Previously this phenomenon occurred in the famous Brouwer's translation theorem, cf. [1]. Here we show that this can already occur in the rigid structure of Lorentz space-forms.

A topological group $G$ is said to act locally properly on a locally compact Hausdorff space $X$ if for every $x \in X$ there exists a neighborhood $U$ s.t. $\{g \in G \mid g U \cap U \neq \varnothing\}$ is relatively compact in $G$. If, moreover $G$ is discrete, the action is said to be locally properly discontinuous. ${ }^{5}$

We shall prove the

Assertion. There exists $\tilde{\Gamma} \approx \mathbf{Z}^{3}$ contained in $I_{0}\left(S_{\infty}\right)$ which acts locally properly discontinuously on $S_{\infty}$. The resulting quotient $M=\tilde{\Gamma} \backslash S_{\infty}$ is an aspherical, noncompact, non-Hausdorff manifold with $\pi_{1}(M) \approx \Gamma \approx \mathbf{Z}^{3}$. It

\footnotetext{
${ }^{5}$ As pointed out in $[11, \S 1]$, the notion "properly discontinuous" is sometimes confused with that of "locally properly discontinuous" in the literature. The words "wandering" or simply "discontinuous" are also sometimes used for the latter notion.
} 
has the weak homotopy type of a 3-torus but does not have a homotopy type of any CW-complex. Topologically, it is a 2-torus bundle over a basic example of a non-Hausdorff 1-manifold, namely, the line with a double point.

We shall call this $M$ a strange space-form. Geometrically it also happens to be a circle bundle over a "strange" 2-dimensional Lorentz space-form of constant curvature 1 and $\pi_{1} \approx \mathbf{Z}^{2}$.

Here is the construction: In the notation of $\S 2$ consider $G=A \times N \subseteq P_{1} \times$ $P_{1}=I_{0}\left(S_{1}\right)$ acting on $S_{1}$. Then $G \approx \mathbf{R}^{2}$ acts locally properly, and any discrete subgroup $\Gamma \approx \mathbf{Z}^{2}$ of $G$ acts locally properly discontinuously. Take $M=\Gamma \backslash S_{1}$ where $\Gamma$ consists of $\mathbf{Z}$-actor in each $A$ and $N$.

Proof (sketch). Let

$$
a=\left[\begin{array}{cc}
\lambda & 0 \\
0 & \lambda^{-1}
\end{array}\right]^{-}, \quad n=\left[\begin{array}{ll}
1 & r \\
0 & 1
\end{array}\right]^{-}, \quad p=\left[\begin{array}{cc}
x & y \\
z & w
\end{array}\right]^{-} .
$$

We have

$$
(a, n) p=a p n^{-1}=\left[\begin{array}{cc}
\lambda x & \lambda(-r x+y) \\
\lambda^{-1} z & \lambda^{-1}(-r z+w)
\end{array}\right]^{-} .
$$

The action is cleariy free since $x, z$ are not simultaneously zero. Moreover, if $p$ and

$$
p^{\prime}=\left[\begin{array}{cc}
x^{\prime} & y^{\prime} \\
z^{\prime} & w^{\prime}
\end{array}\right]^{-}
$$

are close, and $(a, n) p$ and $p^{\prime}$ are close then

$$
\left[\begin{array}{cc}
\lambda x-x^{\prime} & \lambda(-r x+y)-y^{\prime} \\
\lambda^{-1} z-z^{\prime} & \lambda^{-1}(-r z+w)-w^{\prime}
\end{array}\right]^{-} \text {is close to }\left[\begin{array}{ll}
0 & 0 \\
0 & 0
\end{array}\right]^{-} .
$$

One sees easily that $(a, n)$ must be close to identity.

By contrast, recall that $G$ does not act properly, cf. (5.1).

To understand the topology of $M$ consider $P_{1} / N$ as the space of horocycles in the unit disk model $D$ of the hyperbolic plane. Then $P_{1} / N \approx S^{1} \times(0,1)$ where $\theta \in S^{1}$ denotes the point of tangency of the horocycle at $\partial D$ and $r \in(0,1)$ is the Euclidean radius of the horocycle. Notice that $A$ has two fixed points, which we call 0 and $\infty$, on $\partial D$ outside of which $A$ acts freely. The induced action of $a \in A$ on $P_{1} / N \approx S^{1} \times(0,1)$, in the $S^{1}$-factor is the same as that on $\partial D$, and on the second factor it amounts to taking the pencil of horocycles tangent at $p \in \partial D$ to that tangent at $a \cdot p$. This gives a free $A$-action on $P_{1} / N$ and exhibits $P_{1} / N$ as a principle $\mathbf{R}_{+}$-bundle on $X=$ $A \backslash P_{1} / N$. (Here $\mathbf{R}_{+}$is the multiplicative group of positive reals.) The segments $0 \times(0,1)$ and $\infty \times(0,1)$ project onto two distinct distinguished points, say $\overline{0}$ 
and $\infty$ resp., in $X$. On the complement of either of the segments $0 \times(0,1)$ or $\infty \times(0,1)$ in $P_{1} / N$ the $A$-action is proper, and so $X-\overline{0}$ or $X-\bar{\infty}$ is $\approx$ an open interval. But a neighborhood of $\overline{0}$ is obtained from a neighborhood of $\bar{\infty}$, by deleting $\bar{\infty}$, and inserting $\overline{0}$. In other words $X$ is homeomorphic to the line with a double point.

Notice that $\pi_{1}(X) \approx \mathbf{Z}$ but any map of $X$ into a CW-complex is homotopic to a constant. So $X$ has a weak homotopy type of a circle but does not have a homotopy type of any $\mathrm{CW}$-complex,

All the statements about $M$ in the assertion are now clear.

Note that $A \backslash P_{1}$ is a model space for 2-dimensional Lorentz geometry of constant curvature 1 . So $A \backslash P_{1} / \mathbf{Z}$ is a "strange" 2-dimensional Lorentz space-form and, $M$ may be regarded as a circle bundle over this one.

(11.1.1) Remark. The first integral Čech cohomology groups of $X$ and $M$ are a bit weird. They are $\check{H}^{1}(X, \mathbf{Z})=0, \check{H}^{1}(M ; \mathbf{Z}) \approx \mathbf{Z}^{2}$. Also $P_{1} / N$ regarded as a $\mathbf{R}_{+}$-bundle over $X$ is not trivial, but is trivialized on $U=X-\{\overline{0}\}$ and $V=X-\{\bar{\infty}\}$. The covering $\{U, V\}$, however, is not "numerable" in the sense of Dold, cf. [4].

\section{References}

[1] L. E. J. Brouwer, Beweis des ebenen Translationssatzes, Math. Ann. 72 (1912) 37-54.

[2] P. E. Conner \& F. Raymond, Actions of compact Lie groups on aspherical manifolds, Topology of Manifolds, Proc. Summer Institute, University of Georgia, Athens, 1969, Markham, Chicago 1970, 227-264.

[3] __ Deforming homotopy equivalences to homeomorphisms in aspherical manifolds, Bull. Amer. Math. Soc. 83 (1977) 36-85.

[4] A. Dold, Partitions of unity in the theory of fibrations, Ann. of Math. (2) 78 (1963) 223-255.

[5] S. W. Hawking \& G. F. R. Ellis, The large scale structure of space-time, Cambridge Univ. Press, Cambridge, 1973.

[6] J. Hempel, 3-manifolds, Annals of Math. Studies, No. 86, Princeton Univ. Press, Princeton, 1976.

[7] W. Jaco, Lectures on 3-manifold topology, CBMS Reg. Conf. Ser. Math., No. 43, Amer. Math. Soc., Providence, 1980.

[8] R. S. Kulkarni, On manifolds with indefinite metrics, preprint, 1973.

[9] _ On the principle of Uniformization, J. Differential Geometry 13 (1978) 109-138.

[10] _ Fundamental groups of homogeneous space-forms, Math. Ann. 234 (1978) 51-60.

[11] Proper actions and pseudo-Riemannian space-forms, Advances in Math. 40 (1981) $10-51$.

[12] Geometries for 3- and 4-manifolds, in preparation.

[13] R. S. Kulkarni, K. B. Lee \& F. Raymond, Deformation spaces for Seifert manifolds, (to appear) Topology Proc., Special Year, Md., 1983-84 (ed., Jay Alexander and John Harer), Lecture Notes in Math., Springer, New York.

[14] R. S. Kulkarni \& F. Raymond, Three-dimensional Lorentz space forms and Seifert fiber spaces, Differential Geometry Proc., Special Year, Md., 1981-82 (ed., R. Brooks, A. Gray and B. L. Reinhart), Progress in Math., Vol. 32, Birkhäuser, Boston, 1983, 177-188. 
[15] K. B. Lee \& F. Raymond, Geometric realization of group extensions by the Seifert construction, Contemporary Math., Vol. 33, 1984, 353-411.

[16] S. MacLane, Homology, 3rd ed., Springer, Berlin, 1975.

[17] W. Meeks \& G. P. Scott, Finite group actions on 3-manifolds, in preparation.

[18] J. Milnor, On the existence of a connection with curvature zero, Comment. Math. Helv. 32 (1957) 215-223.

[19] On the 3-dimensional Brieskorn manifolds $M(p, q, r)$ in knots, Groups and 3-Manifolds (papers dedicated to R. H. Fox) (ed. L. P. Neuwirth), Annals of Math. Studies, No. 84, 175-225, Princeton Univ. Press, Princeton, 1975.

[20] P. Orlik, Seifert manifolds, Lecture Notes in Math., Vol. 291, Springer, Berlin, 1972.

[21] P. Orlik \& F. Raymond, On 3-manifolds with local SO(2)-action, Quart. J. Math., 20 (1969) 143-160.

[22] P. Orlik \& P.Wagreich, Isolated singularities of algebraic surfaces with $\mathbf{C}^{*}$-action, Ann. of Math. (2) 99 (1971) 205-228.

[23] W. Neumann \& F. Raymond, Seifert manifolds, plumbing, $\mu$-invariant and orientation-reversing maps, Lecture Notes in Math. Vol. 664, Springer, Berlin, 1977, 162-195.

[24] J. C. Oxtoby, Measure and category, 2nd ed., Springer, Berlin, 1980.

[25] R. S. Palais, On the existence of slices for actions of noncompact Lie groups, Ann. of Math. (2) 73 (1961) 295-323.

[26] M. S. Raghunathan, Discrete subgroups of Lie groups, Ergebnisse Math. u. i. Grenzgeb., Vol. 68, Springer, Berlin, 1972.

[27] F. Raymond, Classification of the actions of the circle on 3-manifolds, Trans. Amer. Math. Soc. 31 (1968) 51-78.

[28] F. Raymond \& A. Vasquez, Closed 3-manifolds whose universal covering is a Lie group, Topology Appl. 12 (1981) 161-179.

[29] E. Ruh, On the automorphism group of a G-structure, Comment. Math. Helv. 39 (1964) 189-204.

[30] G. P. Scott, There are no fake Seifert fiber spaces with infinite $\pi_{1}$, Ann. of Math. (2) 117 (1983) 35-70.

[31] The geometries of 3-manifolds, Bull. London Math. Soc. 15 (1983) 401-487.

[32] H. Seifert, Topologie dreidimensionaler gefaserter Räume, Acta Math. 60 (1932) 147-238.

[33] C. L. Siegel, Bemerkung zu einem Satz von Jakob Nielsen, Gesam. Abh. III, 56, 92-96.

[34] W. Thurston, Three-dimensional manifolds, Kleinian groups and hyperbolic geometry, Bull. Amer. Math. Soc. 6 (1982) 357-381.

[35] The geometry and topology of three-manifolds, Lecture Notes, Princeton University.

[36] R. Waldhausen, Gruppen mit Zentrum and 3-dimensionale Mannigfaltigkeiten, Topology 6 (1967) 505-517.

[37] J. Wolf, Homogeneous manifolds of constant curvature, Comment. Math. Helv. 36 (1961) 112-147.

[38] _ Spaces of constant curvature, 3rd ed., Publish or Perish, Boston, 1974.

[39] H. Zieschang, E. Vogt \& H. Coldeway, Surfaces and planar discontinuous groups, Lecture Notes in Math., Vol. 835, Springer, Berlin, 1980. 